Homology, Homotopy and Applications, vol.8(1), 2006, pp.263-292

\title{
A MODEL CATEGORY FOR LOCAL PO-SPACES
}

\section{PETER BUBENIK AND KRZYSZTOF WORYTKIEWICZ}

\author{
(communicated by J.F. Jardine)
}

\begin{abstract}
Locally partial-ordered spaces (local po-spaces) have been used to model concurrent systems. We provide equivalences for these spaces by constructing a model category containing the category of local po-spaces. We show that the category of simplicial presheaves on local po-spaces can be given Jardine's model structure, in which we identify the weak equivalences between local po-spaces. In the process, we give an equivalence between the category of sheaves on a local po-space and the category of étale bundles over a local po-space. Finally, we describe a localization that should provide a good framework for studying concurrent systems.
\end{abstract}

\section{Introduction}

The motivation for this paper stems from the study of concurrent processes accessing shared resources. Such systems were originally described by discrete models based on graphs, possibly equipped with some additional information [Mil80]. The precision of these models suffers, however, from an inaccuracy in distinguishing between concurrent and non-deterministic executions. It turned out that a satisfactory way to organize this information can be based on cubical sets, giving rise to the notion of Higher-Dimensional Automata or HDA's [Gou96, Gou02]. HDA's live in slice categories of $\mathbf{c S e t}$, the category of cubical sets and their morphisms.

A different view, which has its origins in Dijkstra's notion of progress graphs [Dij68], takes the flow of time into account. The difficulty here is to adequately model the fact that time is irreversible as far as computation is concerned. On the other hand, one would like to identify execution paths corresponding to (at least) the same sequence of acquisitions of shared resources. However, in order not to lose precision, this notion of homotopy is also subject to the constraint above of the irreversibility of time. There are two distinct approaches, both based on topological spaces.

One approach, advocated by Gaucher, is to topologize the sets of paths between the states of an automaton, which technically amounts to an enrichment with no

This research was partially funded by the Swiss National Science Foundation grant 200020-105383. Received June 17, 2005, revised January 10, 2006; published on February 10, 2006.

2000 Mathematics Subject Classification: Primary 55U35, 18G55, 68Q85; Secondary 18F20, 55U10. Key words and phrases: local po-spaces (local pospaces), abstract homotopy theory, model categories, concurrency, simplicial presheaves, sheaves, étale bundles, directed homotopy (dihomotopy), context.

Copyright (C) 2006, International Press. Permission to copy for private use granted. 
units [Gau03]. The intuition behind the setup is to distinguish between spatial and temporal deformations of computational paths. The related framework of Flows has clear technical advantages from a (model-)categorical point of view.

The other approach, advocated by Fajstrup, Goubault, Raussen and others, is to topologize partially ordered states of automata. Such objects are called partiallyordered spaces or po-spaces (also pospaces) ${ }^{1}$. The advantage of using po-spaces is that there is a very simple and intuitive way to express directed homotopy or dihomotopy [Gou03, FGR99].

However, the price paid is that po-spaces cannot model executions of (concurrent) programs with loops. The solution is to order the underlying topological space only locally. Such objects are called local po-spaces and the notion of dihomotopy becomes more intricate in this context. Nevertheless, practical reasons like tractability call for a good notion of equivalence in the category of local po-spaces. Put differently, it would be useful to be able to replace a given local po-space model with a simpler local po-space which nevertheless preserves the relevant computer-scientific properties.

In this paper, we study these questions in the framework of Quillen's (closed) model categories [Qui67, Hov99, Hir03]. Briefly, a model category is a category with all small limits and colimits and three distinguished classes of morphisms called weak equivalences, cofibrations and fibrations. Weak equivalences that are also cofibrations or fibrations are called trivial cofibrations and trivial fibrations, respectively. These morphisms satisfy four axioms that allow one to apply the machinery of homotopy theory to the category. This machinery allows a rigorous study of equivalences. We remark that there are other frameworks for studying equivalence. However, model categories have the most developed theory, and have succeeded in illuminating many diverse subjects.

Our aim is to construct a model category of locally partial-ordered spaces as a foundation for the study of concurrent systems. This is technically difficult because locally partial-ordered spaces are not known to be closed under taking colimits. We will define a category LPS of local po-spaces, which embeds into the category sPre(LPS) of simplicial presheaves on local po-spaces. The objects of $\mathbf{s P r e}(\mathbf{L P S})$ are contravariant functors from LPS to the category of simplicial sets and the morphisms are the natural transformations. This embedding is given by a Yoneda embedding (see Definition 2.17),

$$
\bar{y}: \text { LPS } \rightarrow \text { sPre(LPS). }
$$

We now briefly describe some technical conditions on model categories which strengthen our theorems. For more details, see Definitions 8.2 and 8.4 and [Hov99, Hir03]. A model category is proper if the weak equivalences are closed under both pushouts with cofibrations and pullbacks with fibrations. It is left proper if the first condition holds. A model category is cofibrantly generated if the model category structure is induced by a set of generating cofibrations and a set of generating trivial cofibrations, both of which permit the small object argument. A cellular model category is a cofibrantly generated model category in which the cell complexes are

${ }^{1}$ Grandis uses a related approach [Gra03] in which the underlying topological space comes with a class of directed paths. However, these spaces are not partially-ordered, even locally. 
well behaved. A simplicial model category $\mathcal{M}$ is a model category enriched over simplicial sets, which for any $X \in \mathcal{M}$ and any simplicial set $K$ has objects $X \otimes K$ and $X^{K}$ which satisfy various compatibility conditions.

Theorem 1.1. The category $\mathbf{s P r e ( L P S ) ~ h a s ~ a ~ p r o p e r , ~ c e l l u l a r , ~ s i m p l i c i a l ~ m o d e l ~}$ structure in which

- the cofibrations are the monomorphisms,

- the weak equivalences are the stalkwise equivalences, and

- the fibrations are the morphisms which have the right lifting property with respect to all trivial cofibrations.

Furthermore among morphisms coming from LPS (using the Yoneda embedding $\mathbf{L P S} \hookrightarrow \mathbf{s P r e}(\mathbf{L P S}))$, the weak equivalences are precisely the isomorphisms.

The model structure on sPre(LPS) is Jardine's model structure [Jar87, Jar96] on the category of simplicial presheaves on a small Grothendieck site. We show that Shv(LPS) is a Grothendieck topos which has enough points. Under this condition, Jardine showed that the weak equivalences are the stalkwise equivalences.

This model category can be thought of as a localization of the universal injective model category of local po-spaces [Joy84, Dug01, DHI04]. While, in general, the weak equivalences are interesting and non-trivial [Jar87], this is not true for those coming from LPS. To obtain a more interesting category from the point of view of concurrency, we would like to localize with respect to directed homotopy equivalences. In [Bub04], it is argued that the relevant equivalences are the directed homotopy equivalences relative to some context. The context is a local po-space $A$ and the directed homotopy equivalences rel $A$ are a set of morphisms in A $\downarrow$ LPS.

We combine this approach with Theorem 1.1 as follows. First, we remark that $A$ embeds in sPre(LPS) as $\bar{y}(A)$. Next, the model structure on sPre(LPS) induces a model structure on the coslice category $\overline{\mathbf{y}}(\mathbf{A}) \downarrow \mathbf{s P r e}(\mathbf{L P S})$. Finally, one can take the left Bousfield localization of this model category with respect to the directed homotopy equivalences rel $A$.

Theorem 1.2. Let $\mathcal{I}=\{\bar{y}(f) \mid f$ be a directed homotopy equivalence rel $A\}$. Then the category $\overline{\mathbf{y}}(\mathbf{A}) \downarrow \mathbf{s P r e}(\mathbf{L P S})$ has a left proper, cellular model structure in which

- the cofibrations are the monomorphisms,

- the weak equivalences are the I-local equivalences, and

- the fibrations are those morphisms which have the right lifting property with respect to monomorphisms which are $\mathcal{I}$-local equivalences.

Recall that, given a topological space $Z$, étale bundles over $Z$ are maps $W \rightarrow Z$ which are local homeomorphisms. Let $\mathcal{O}(Z)$ be $Z$ 's locale of open subsets and recall that sheaves over $Z$ are functors $\mathcal{O}(Z)^{o p} \rightarrow$ Set that enjoy a good gluing property. There is a well-known correspondence between étale bundles and sheaves. We establish a directed version of this correspondence, which may be of independent interest. 
Theorem 1.3. Let $Z \in \mathbf{L P S}$. Let $\operatorname{Etale}(Z)$ be the category of di-étale bundles over $Z$, i.e. the category of bundles which are local dihomeomorphisms. Let $\mathcal{O}(Z)$ be the category of open subobjects of $Z$. There is an equivalence of categories:

$$
\Gamma: \operatorname{Etale}(Z) \leftrightarrows \operatorname{Shv}(\mathcal{O}(Z)): \Lambda
$$

\section{Acknowledgements}

The authors would like to thank Eric Goubault, Emmanuel Haucourt, Kathryn Hess and Phil Hirschhorn for helpful discussions and suggestions.

\section{Contents}

2 Background $\quad 266$

3 Local po-spaces $\quad 273$

4 The open-dicover topology 275

$5 \quad$ Equivalence of sheaves and di-étale bundles $\quad 277$

6 Points 280

7 Stalkwise Equivalences $\quad 282$

8 Model Categories for Local Po-spaces $\quad 284$

$\begin{array}{lr}\text { A Hypercovers } & 289\end{array}$

\section{Background}

This section contains some known definitions and facts we build on. We start by stating the definition of a model category in Section 2.1. Next, we review the basics on presheaves in Section 2.2 and on sheaves in Section 2.3. We then recall the notions of topoi and geometric morphisms in Section 2.4 and of stalks in Section 2.5. Our main reference for this material is [MLM92]. Section 2.6 is devoted to some important model structures on $\mathbf{s S e t}^{\mathbb{C}^{o p}}$, the category of simplicial presheaves over a category $\mathbb{C}$. The material is drawn from [Jar87, Jar96, DHI04].

\subsection{Model categories}

Recall that a morphism $i: A \rightarrow B$ has the left lifting property with respect to a morphism $p: X \rightarrow Y$ if in every commutative diagram 


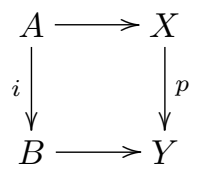

there is a morphism $h: B \rightarrow X$ making the diagram commute. Also, $f$ is a retract of $g$ if there is a commutative diagram:

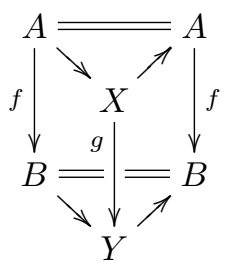

Definition 2.1. A model category is a category with all small limits and colimits that has three distinguished classes of morphisms: $\mathcal{W}$, called the weak equivalences; $\mathcal{C}$, called the cofibrations; and $\mathcal{F}$, called the fibrations, which together satisfy the axioms below. We remark that morphisms in $\mathcal{W} \cap \mathcal{C}$, and $\mathcal{W} \cap \mathcal{F}$, are called trivial cofibrations and trivial fibrations, respectively.

1. Given composable morphisms $f$ and $g$ if any of the two morphisms $f, g$, and $g \circ f$ are in $\mathcal{W}$, then so is the third.

2. If $f$ is a retract of $g$ and $g$ is in $\mathcal{W}, \mathcal{C}$ or $\mathcal{F}$, then so is $f$.

3. Cofibrations have the left-lifting property with respect to trivial fibrations, and trivial cofibrations have the left-lifting property with respect to fibrations.

4. Every morphism can be factored as a cofibration followed by a trivial fibration, and as a trivial cofibration followed by a fibration. These factorizations are functorial.

\subsection{Presheaves}

Recall that a presheaf $P$ on $\mathbb{C}$ is just a functor $P \in \mathbf{S e t}^{\mathbb{C}^{o p}}$. In particular, "homing"

$$
\begin{array}{llll}
\mathbb{C}\left({ }_{-}, C\right): & \mathbb{C}^{o p} & \rightarrow & \text { Set } \\
& X & \mapsto \mathbb{C}(X, C)
\end{array}
$$

gives rise to a presheaf and further to the Yoneda embedding

$$
\begin{aligned}
y: \quad \mathbb{C} & \mapsto \text { Set }^{\mathbb{C}^{o p}} \\
C & \mapsto \mathbb{C}\left({ }_{-}, C\right) .
\end{aligned}
$$

This embedding is dense, i.e.

$$
P \cong \operatorname{colim}(y \circ \pi)
$$

canonically for any presheaf $P$, where $\pi:(y \downarrow P) \rightarrow \mathbb{C}$ is the projection from the comma-category $y \downarrow P$. Recall that a presheaf in the image of the Yoneda-embedding (up to equivalence) is called representable. 


\subsection{Sheaves}

Definition 2.2. A sieve on $M \in \mathbb{C}$ is a subfunctor $S \subseteq \mathbb{C}\left({ }_{-}, M\right)$. A Grothendieck topology $J$ on $\mathbb{C}$ assigns to each $M \in \mathbb{C}$ a collection $J(M)$ of sieves on $M$ such that

(i) (maximal sieve) $\mathbb{C}\left({ }_{-}, M\right) \in J(M)$ for all $M \in \mathbb{C}$;

(ii) (stability under pullback) if $g: M \rightarrow N$ and $S \in J(N)$, then $\left(g \circ{ }_{-}\right)^{*}(S) \in J(M)$ as given by

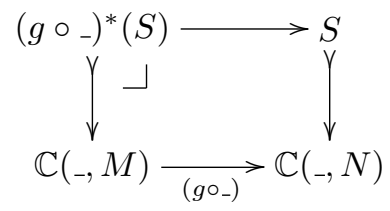

(iii) (transitivity) if $S \in J(M)$ and $R$ is a sieve on $M$ such that $\left(f \circ \circ_{-}\right)^{*}(R) \in J(U)$ for all $f: U \rightarrow M$ in the image of $S$, then $R \in J(M)$;

We say that a sieve $S$ on $M$ is a covering sieve or a cover of $M$ whenever $S \in J(M)$.

Remark 2.3. Unwinding Definition 2.2 pinpoints a sieve as a right ideal, i.e. a set of arrows $S$ with codomain $M$ such that $f \in S \Longrightarrow f \circ h \in S$ whenever the codomain of $h, \operatorname{cod}(h)=\operatorname{dom}(f)$, the domain of $f$. From this point of view, pulling back a sieve $S$ on $M$ by an arrow $N \stackrel{f}{\rightarrow} M$ amounts to building the set

$$
f^{*}(S) \stackrel{\text { def }}{=}\{h \mid \operatorname{cod}(h)=N, f \circ h \in S\} .
$$

It is then immediate how to rephrase a Grothendieck topology in terms of right ideals.

Definition 2.4. Let $J$ be a Grothendieck topology on $\mathbb{C}$. A presheaf $P \in \operatorname{Set}^{\mathbb{C}^{o p}}$ is a sheaf with respect to $J$ provided any natural transformation $\theta: S \Rightarrow P$ uniquely extends through $y(M)$ as in

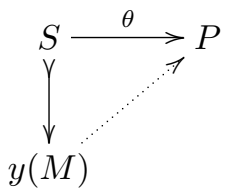

for all $S \in J(M)$ and all $M \in \mathbb{C} . J$ is subcanonical if the representable presheaves are sheaves.

Remark 2.5. Let $\theta: S \rightarrow P$ be a natural transformation from a sieve $S$ to a presheaf $P$. If one sees $S$ as a right ideal $S=\left\{u_{j}: M_{j} \rightarrow M\right\}$, then $\theta$ amounts to a function 
that assigns to every $u_{j}: M_{j} \rightarrow M \in S$ an element $a_{j} \in P\left(M_{j}\right)$ such that

$$
P(v)\left(a_{j}\right)=a_{k}
$$

for all $v: M_{k} \rightarrow M_{j}$ and for all $u_{k}=u_{j} \circ v \in S$. Such a function is called a matching family for $S$ of elements of $P$. A matching family $a_{j} \in P\left(M_{j}\right)$ admits an amalgamation $a \in P(M)$ if

$$
P\left(u_{j}\right)(a)=a_{j}
$$

for all $u_{j}: M_{j} \rightarrow M \in S$. From this point of view, the Yoneda Lemma characterizes a sheaf as a presheaf such that every matching family has a unique amalgamation for all $S \in J(M)$ and all $M \in \mathbb{C}$.

A Grothendieck topology is a huge object. In practice, a generating device is used.

Definition 2.6. A basis $K$ for a Grothendieck topology assigns to each object $M$ a collection $K(M)$ of families of morphisms with codomain $M$ such that

(i) all isomorphisms $f: U \rightarrow M$ are contained in $K(M)$,

(ii) given a morphism $g: N \rightarrow M \in \mathbb{C}$ and $\left\{f_{i}: U_{i} \rightarrow M\right\} \in K(M)$, then the family of pullbacks $\left\{\pi_{2}: U_{i} \times_{M} N \rightarrow N\right\} \in K(N)$, and

(iii) given $\left\{f_{i}: U_{i} \rightarrow M\right\} \in K(M)$ and for each $i,\left\{h_{i j}: A_{i j} \rightarrow U_{i}\right\} \in K\left(U_{j}\right)$, then the family of composites $\left\{f_{i} \circ h_{i j}: A_{i j} \rightarrow M\right\} \in K(M)$.

Remark 2.7. Given a basis $K$ for a Grothendieck topology, one generates the corresponding Grothendieck topology $J$ by defining

$$
V \in J(M) \Longleftrightarrow \text { there is } U \in K(M) \text { such that } U \subset V .
$$

As expected, the sheaf condition can be rephrased in terms of a basis.

As an example, consider the case $\mathbb{C}=\mathcal{O}(X)$ with $X$ a topological space and $\mathcal{O}(X)$ its locale of opens. The basis of the open-cover (Grothendieck) topology is, as expected, given by open coverings of the opens.

Theorem 2.8. Let $\mathbf{S h v}(\mathbb{C}, J)$ be the full subcategory of $\mathbf{S e t}^{\mathbb{C}^{o p}}$ whose objects are sheaves for $J$. The inclusion functor $i: \operatorname{Shv}(\mathbb{C}, J) \rightarrow \mathbf{S e t}^{\mathbb{C}^{\mathrm{p}}}$ has a left adjoint a called the associated sheaf functor or sheafification. This left adjoint preserves finite limits.

Theorem 2.8 is listed as Theorem III.5.1 in [MLM92]. There are several equivalent ways to construct the associated sheaf functor, the most classical one being the "plus-construction" applied twice.

Remark 2.9. A cover on $M$ amounts to a cocone in $\mathbb{C}$ with vertex $M$. The associated sheaf functor maps these cocones onto colimiting ones. Moreover, it is universal with respect to this property. 


\subsection{Topoi}

Definition 2.10. A category $\mathcal{E}$ has exponentials provided that for all $X \in \mathcal{E}$, the functor ${ }_{-} \times X: \mathcal{E} \rightarrow \mathcal{E}$ has a right adjoint denoted $\left({ }_{-}\right)^{X}$, so that

$$
\mathcal{E}(Y \times X, Z) \cong \mathcal{E}\left(Y, Z^{X}\right) .
$$

Suppose now $\mathcal{E}$ has a terminal object 1 , and has finite limits. A subobject classifier is a monomorphism true: $1 \longmapsto \Omega$ such that for every monomorphism $s: S \longmapsto X$, there is a unique morphism $\phi_{S}$ such that pullback of true along $\phi_{S}$ yields $s$ :

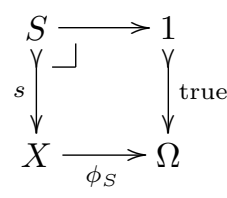

The category $\mathcal{E}$ is a topos if it has exponentials and a subobject classifier.

A subobject classifier is obviously unique (up to isomorphism). Furthermore, a topos has all finite colimits, though this is not easy to prove. It would take pages to enumerate all the remarkable features of a topos, see [Joh77] for an introduction to the lore of the material. Let us just say that topoi as introduced by Grothendieck and his collaborators had a very strong algebro-geometrical flavor [AGV72], yet the rich structure is relevant not only for algebraic geometers, but for logicians as well [Law63, Law64, Law73].

Definition 2.11. A site $(\mathbb{C}, J)$ is a small category $\mathbb{C}$ equipped with a Grothendieck topology $J$. A Grothendieck topos is a category equivalent to the category $\operatorname{Shv}(\mathbb{C}, J)$ of sheaves on $(\mathbb{C}, J)$.

The following are well known.

Proposition 2.12. 1. A Grothendieck topos is a topos;

2. Set is a topos;

3. Set ${ }^{\mathbb{C o p}}$ is a topos for any $\mathbb{C}$.

Definition 2.13. Let $\mathcal{E}$ and $\mathcal{F}$ be topoi. A geometric morphism $g: \mathcal{F} \rightarrow \mathcal{E}$ is a pair of adjoint functors

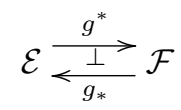

such that the left adjoint $g^{*}$ is left-exact (that is, it preserves finite limits). The right adjoint is called direct image and the left one inverse image.

As an example, $i: \mathbf{S h v}(\mathbb{C}, J) \hookrightarrow \mathbf{S e t}^{\mathbb{C}^{o p}}$ is the direct image part of a geometric morphism. Notice that the convention for a geometric morphism is to have the direction of its direct image part. 
Definition 2.14. A (geometric) point in a topos $\mathcal{E}$ is a geometric morphism

$$
p: \text { Set } \rightarrow \mathcal{E}
$$

(we write $p \in \mathcal{E}$ by abuse of notation). A topos $\mathcal{E}$ has enough points if given $f \neq$ $g: P \rightarrow Q \in \mathcal{E}$, there is a point $p \in \mathcal{E}$ such that $p^{*} f \neq p^{*} g \in$ Set.

\subsection{Stalks and Germs}

Definition 2.15. Let $(\mathbb{C}, J)$ be a site, $a$ : $\operatorname{Set}^{\mathbb{C}^{o p}} \rightarrow \operatorname{Shv}(\mathbb{C}, J)$ the associated sheaf functor and $x \in \operatorname{Shv}(\mathbb{C}, J)$ a point. The stalk functor at $x$ is given by

$$
\text { stalk }_{x} \stackrel{\text { def }}{=} x^{*} \circ a: \text { Set }^{\mathbb{C}^{o p}} \rightarrow \text { Set. }
$$

Given a presheaf $F$, we say that $\operatorname{stalk}_{x}(F)$ is the stalk of $F$ at $x$. As an example, consider again the case $\mathbb{C}=\mathcal{O}(X)$ with $X$ a (this time) Hausdorff topological space and $\mathcal{O}(X)$ its locale of opens equipped with the open-cover topology. Let $\operatorname{Shv}(X)$ be the corresponding topos of sheaves. It can be shown that any geometric point $x: \mathbf{S e t} \rightarrow \mathbf{S h v}(X)$ corresponds to a "physical" point $x^{\prime} \in X$. The stalk of $F \in \operatorname{Set}^{\mathcal{O}(X)^{\text {op }}}$ at $x$ is then given by

$$
\operatorname{stalk}_{x}(F):=\underset{U \in \mathcal{O}(X), x^{\prime} \in U}{\operatorname{colim}} F(U) .
$$

Write $\operatorname{germ}_{x, U}: F(U) \rightarrow \operatorname{stalk}_{x}(F)$ for the canonical map at $U$ (germ $\operatorname{gen}_{x}$ when $U$ is clear from the context). We call the equivalence class $\operatorname{germ}_{x, U}(s)$ of $s$ in $\operatorname{stalk}_{x}(F)$ the germ of $s$ at $x$. Obviously,

$$
\operatorname{stalk}_{x}(F)=\left\{\operatorname{germ}_{x, U}(s) \mid U \in \mathcal{O}(X), x^{\prime} \in U, s \in F(U)\right\} .
$$

\subsection{Simplicial Presheaves}

For the rest of this section, let $\mathbb{C}$ be a small category with a Grothendieck topology $J$ such that $\operatorname{Shv}(\mathbb{C}, J)$ has enough points.

Let $\Delta$ be the simplicial category which has objects $[n]=\{0,1, \ldots, n\}$ for $n \geqslant 0$, and whose morphisms are the maps such that $x \leqslant y$ implies that $f(x) \leqslant f(y)$. Then sSet is the category $\mathbf{S e t}^{\Delta^{\mathrm{op}}}$. This category has a well-known model structure (e.g. see [Hov99]) where $\mathcal{W}_{\mathbf{s S e t}}$ are the morphisms whose geometric realization is a weak homotopy equivalence and $\mathcal{C}_{\text {sSet }}$ are the monomorphisms.

Objects of $\mathbf{s S e t}^{\mathbb{C}^{\text {op }}}$ are called simplicial presheaves on $\mathbb{C}$ since

$$
\mathbf{S S e t}^{\mathbb{C}^{o p}}=\left(\operatorname{Set}^{\Delta^{o p}}\right)^{\mathbb{C}^{o p}} \cong \operatorname{Set}^{\Delta^{o p} \times \mathbb{C}^{o p}} \cong\left(\operatorname{Set}^{\mathbb{C}^{o p}}\right)^{\Delta^{o p}}
$$

There is an embedding

$$
\begin{aligned}
\kappa: \text { Set }^{\mathbb{C}^{o p}} & \rightarrow \text { sSet }^{\mathbb{C}^{o p}} \\
F & \mapsto \kappa_{F},
\end{aligned}
$$

where $\kappa_{F}$ is constant levelwise i.e. $\left(\kappa_{F}\right)(C)_{n} \stackrel{\text { def }}{=} F(C)$ for all $n \in \mathbb{N}$, and all the face and degeneracy maps are the identity. There is a further embedding

$$
\begin{aligned}
\gamma: \mathbf{s S e t} & \rightarrow \text { sSet }^{\mathbb{C}^{o p}} \\
K & \mapsto \gamma_{K},
\end{aligned}
$$


where $\gamma_{K}$ is constant objectwise i.e. $\gamma_{K}(C) \stackrel{\text { def }}{=} K$ for all $C \in \mathbb{C}$.

Recall that for $C \in \mathbb{C}$ and $F \in$ Set $^{\mathbb{C}^{\text {op }}}$, the Yoneda Lemma gives the isomorphism Set $^{\mathbb{C}^{\text {op }}}(y(C), F) \cong F(C)$, where $y$ is the Yoneda embedding (see Section 2.2). In the simplicial case, we have the following variation, which can be proved using the same idea used in the proof of the Yoneda Lemma.

Proposition 2.16. (Bi-Yoneda) Let $C \in \mathbb{C}$ and $F \in$ sSet $^{\mathbb{C}^{o p}}$. There is an isomorphism

$$
\operatorname{sSet}^{\mathbb{C}^{o p}}\left(\kappa_{y(C)} \times \gamma_{\Delta[n]}, F\right) \cong F(C)_{n},
$$

natural in all variables.

Definition 2.17. Using the Yoneda embedding $y: \mathbb{C} \rightarrow$ Set $^{\mathbb{C}^{o p}}$ for presheaves one can define an embedding

$$
\bar{y}: \mathbb{C} \stackrel{y}{\rightarrow} \operatorname{Set}^{\mathbb{C}^{\text {op }}} \stackrel{\kappa}{\longrightarrow} \mathbf{s S e t}^{\mathbb{C}^{\text {op }}}
$$

for simplicial presheaves. The functor $\bar{y}$ is also called a Yoneda embedding.

There are two Quillen equivalent model structures on $\mathbf{s S e t}^{\mathbb{C}^{o p}}$ which are in a certain sense objectwise:

- the projective model structure sSet $\mathbb{C}_{p r j}^{\mathbb{C}^{o p}}$ where $\mathcal{W}_{p r j}$ and $\mathcal{F}_{\text {prj }}$ are objectwise (that is, $f: P \rightarrow Q \in \mathcal{W}_{\text {prj }}\left(\mathcal{F}_{\text {prj }}\right)$ if and only if for all $C \in \mathbb{C}, f(C): P(C) \rightarrow$ $\left.Q(C) \in \mathcal{W}_{\text {sSet }}\left(\mathcal{F}_{\text {sSet }}\right)\right)$, and

- the injective model structure $\mathbf{s} \mathbf{S e t}_{i n j}^{\mathbb{C}^{o p}}$ where $\mathcal{W}_{i n j}$ and $\mathcal{C}_{i n j}$ are objectwise.

These were studied by Bousfield and Kan [BK72] and Joyal [Joy84], respectively.

Proposition 2.18. Both $\mathbf{s S e t}_{p r j}^{\mathbb{C}^{o p}}$ and $\mathbf{s}$ Set $_{i n j}^{\mathbb{C}^{o p}}$ are proper, simplicial, cellular model categories. All objects are cofibrant in the latter. The identity functor is a left Quillen equivalence from the projective model structure to the injective model structure.

The injective one is more handy when it comes down to calculating homotopical localizations, yet the fibrant objects are easier to grasp in the projective one ${ }^{2}$.

Using the stalk functor for presheaves, one can define a simplicial stalk functor for simplicial presheaves.

Definition 2.19. The simplicial stalk functor at a point $p$ in $\mathbf{S h v}(\mathbb{C})$ is given by

$$
\begin{aligned}
()_{p}: \quad \text { sSet }^{\mathbb{C}^{\text {op }}} & \rightarrow \text { sSet } \\
P & \mapsto\left\{\text { stalk }_{p}\left(P_{n}\right)\right\}_{n \geqslant 0} .
\end{aligned}
$$

A morphism $f: P \rightarrow Q \in \mathbf{s S e t}^{\mathbb{C}^{\text {op }}}$ is a stalkwise equivalence if $f_{p}: P_{p} \rightarrow Q_{p} \in \mathbf{s S e t}$ is a weak equivalence for all points $p$ in $\operatorname{Shv}(\mathbb{C})$.

Jardine [Jar87] proved the existence of a local version of Joyal's injective model structure. Since we will only be interested in the special case where $\operatorname{Shv}(\mathbb{C})$ has enough points, we will not recall the definition of local weak equivalences.

${ }^{2}$ They are objectwise Kan. 
Theorem 2.20 ([Jar87, Jar96]). Let $\mathbb{C}$ be a small category with a Grothendieck topology. Then $\mathbf{s S e t}^{\mathbb{C}}{ }^{\mathrm{op}}$ the category of simplicial presheaves on $\mathbb{C}$ has a proper, simplicial, cellular model structure in which

- the cofibrations are the monomorphisms, i.e. the levelwise monomorphisms of presheaves,

- the weak equivalences are the local weak equivalences, and

- the fibrations are the morphisms which have the right lifting property with respect to all trivial cofibrations.

Furthermore, if the Grothendieck topos $\mathbf{S h v}(\mathbb{C})$ has enough points, then the local weak equivalences are the stalkwise equivalences.

Jardine's model structure can be seen to be cellular since it can also be constructed as a left Bousfield localization of the injective model structure [DHI04].

\section{Local po-spaces}

The focus of this section is to provide the reader with the main definitions and constructions. We define a small category of local po-spaces LPS and state some of the properties, most of which are proved in the later sections. We show that Theorem 1.1 follows from these properties and a theorem of Jardine.

To simplify the analysis, we will only work with topological spaces which are subspaces of $\mathbb{R}^{n}$ for some $n$, since this provides more than enough generality for studying concurrent systems. The main technical advantage of this setting is that we obtain small categories.

Definition 3.1. (i) Let Spaces be the category whose objects are subspaces of $\mathbb{R}^{n}$ for some $n$, and whose morphisms are continuous maps.

(ii) Let PoSpaces be the category whose objects are po-spaces: that is $U \in$ Spaces together with a partial order (a reflexive, transitive, anti-symmetric relation) $\leqslant$ such that $\leqslant$ is a closed subset of $U \times U$ in the product topology.

(iii) For any $M \in$ Spaces define an order-atlas on $M$ to be an open $\operatorname{cover}^{3} U=\left\{U_{i}\right\}$ of $M$ indexed by a set $I$, where $U_{i} \in$ PoSpaces. These partial orders are compatible: $\leqslant_{i}$ agrees with $\leqslant_{j}$ on $U_{i} \cap U_{j}$ for all $i, j \in I$. We will usually omit the index set from the notation.

(iv) Let $U$ and $U^{\prime}$ be two order atlases on $M$. Say that $U^{\prime}$ is a refinement of $U$ if for all $U_{i} \in U$, and for all $x \in U_{i}$, there exists a $U_{j}^{\prime} \in U^{\prime}$ such that $x \in U_{j}^{\prime} \subseteq U_{i}$ and for all $a, b \in U_{j}^{\prime}, a \leqslant_{j^{\prime}} b$ if and only if $a \leqslant_{i} b$.

(v) Say that two order atlases are equivalent if they have a common refinement. This is an equivalence relation: reflexivity and symmetry follow from the definition. For transitivity, if $U$ and $U^{\prime}$ have a refinement $V=\left\{V_{i}\right\}$ and $U^{\prime}$ and $U^{\prime \prime}$ have a refinement $W=\left\{W_{j}\right\}$, let $T=\left\{V_{i} \cap W_{j}\right\}$. One can check that $T$ is an order atlas of $M$ and that is a refinement of $U^{\prime}$ and $U^{\prime \prime}$.

\footnotetext{
${ }^{3}$ That is, for all $i, U_{i}$ is open as a subspace of $M$ and $M=\cup_{i} U_{i}$.
} 
Any po-space $(U, \leqslant)$ is a local po-space with the equivalence class of order atlases generated by the order atlas $\{U\}$. As a further example, we remark that any discrete space has a unique equivalence class of order-atlases.

Definition 3.2. Let LPS be the category of local po-spaces described as follows. The objects, called local po-spaces, are all pairs $(M, \mathcal{U})$ where $M$ is an object in Spaces and $\mathcal{U}$ is an equivalence class of order-atlases of $M$. The morphisms, called dimaps are described as follows. $f \in \operatorname{LPS}((M, \mathcal{U}),(N, \mathcal{V}))$ if and only if $f \in \operatorname{Spaces}(M, N)$ and for all $V=\left\{V_{j}\right\}_{j \in J} \in \mathcal{V}$, there is a $U=\left\{U_{i}\right\}_{i \in I} \in \mathcal{U}$ such that for all $i \in I, j \in J$, for all $x, y \in U_{i} \cap f^{-1}\left(V_{j}\right)$,

$$
x \leqslant_{U_{i}} y \Longrightarrow f(x) \leqslant_{V_{j}} f(y) .
$$

Remark 3.3. This condition is not necessarily true for arbitrary $U \in \mathcal{U}$. For example, take $M=\{-1,1\}$ with $\mathcal{U}$ the unique equivalence class of order atlases generated by the order atlas $U=\{\{-1\},\{1\}\}$. Let $f=\operatorname{Id}_{M}:(M, \mathcal{U}) \rightarrow(M, \mathcal{U})$. Now let $M_{+}$be the po-space on $M$ with the ordering $-1 \leqslant 1$ and let $M_{-}$be the po-space on $M$ with the ordering $1 \leqslant-1$. Then $\left\{M_{+}\right\} \in \mathcal{U}$ and $\left\{M_{-}\right\} \in \mathcal{U}$ (both have $U$ as a common refinement). However, even though $-1,1 \in M_{+} \cap f^{-1}\left(M_{-}\right)$,

$$
-1 \leqslant M_{+} 1 \text {, but } f(-1) \not_{M_{-}} f(1) \text {. }
$$

Remark 3.4. It is easy to check that a dimap of po-spaces is also a dimap of local po-spaces. Thus PoSpaces the category of po-spaces is a subcategory of LPS.

\section{Remark 3.5. Subobjects in LPS.}

If $(M, \mathcal{U}) \in \mathbf{L P S}$, then a subspace $L \subseteq M \in$ Spaces inherits local po-space structure as follows. Let $U=\left\{U_{i}\right\} \in \mathcal{U}$ and let $W=\left\{W_{i}\right\}$ where $W_{i}=L \cap U_{i}$ and $W_{i}$ has the partial order inherited from $U_{i}$. Then $W$ is an open cover of $L$ and the partial orders are compatible. That is, $W$ is an order atlas. Let $\mathcal{W}$ be the equivalence class of $W$.

We claim that $\mathcal{W}$ does not depend on the choice of $U$. Let $\tilde{U}=\left\{\tilde{U}_{i}\right\} \in \mathcal{U}$, let $\tilde{W}_{i}=L \cap \tilde{U}_{i}$, and let $\tilde{W}=\left\{\tilde{W}_{i}\right\} . U$ and $\tilde{U}$ have a common refinement $\hat{U}=\left\{\hat{U}_{i}\right\}$. Let $\hat{W}_{i}=L \cap \hat{U}_{i}$ and let $\hat{W}=\left\{\hat{W}_{i}\right\}$. Then one can check that $\hat{W}$ is a common refinement of $W$ and $\tilde{W}$. So the equivalence class of $\tilde{W}$ is also $\mathcal{W}$.

Next, we claim that there is a dimap $\iota:(L, \mathcal{W}) \rightarrow(M, \mathcal{U})$ given by the inclusion $\iota: L \hookrightarrow M$. Let $U=\left\{U_{k}\right\} \in \mathcal{U}$, let $W_{k}=L \cap U_{k}$, and let $W=\left\{W_{k}\right\}$. Then $W \in \mathcal{W}$. Let $x, y \in W_{j} \cap \iota^{-1}\left(U_{k}\right)=W_{j} \cap L \cap U_{k}=W_{j} \cap W_{k}$. Note that $\iota(x)=x$ and $\iota(y)=$ $y$. Then

$$
x \leqslant_{W_{j}} y \Longleftrightarrow x \leqslant_{W_{k}} y \Longleftrightarrow x \leqslant_{U_{k}} y .
$$

Therefore, when $L \subseteq M \in$ Spaces, then there is an induced inclusion $(L, \mathcal{W}) \subseteq$ $(M, \mathcal{U}) \in \mathbf{L P S}$.

The remark above will be used implicitly and without reference in Section 6 .

Definition 3.6. A collection of dimaps $\left\{\phi_{j}:\left(M_{j}, \mathcal{U}^{j}\right) \rightarrow(M, \mathcal{U})\right\}$ LPS is an open dicover if

(i) $\left\{\phi_{j}: M_{j} \rightarrow M\right\}$ is an open cover, and 
(ii) for each $j, \mathcal{U}^{j}$ is the local po-space structure inherited from $(M, \mathcal{U})$.

Remark 3.7. The local po-space structures inherited by the subspaces of $(M, \mathcal{U})$ are compatible. So if $\left\{\phi_{j}:\left(M_{j}, \mathcal{U}^{j}\right) \rightarrow(M, \mathcal{U})\right\}$ is an open cover, then for each $j$, there is a $U^{j}=\left\{U_{k}^{j}\right\} \in \mathcal{U}^{j}$ such that $U^{\prime}=\left\{U_{k}^{j}\right\}_{j, k}$ is an order atlas for $M$ and $U^{\prime} \in \mathcal{U}$.

The following is easy to check.

Lemma 3.8. Spaces and LPS are small categories.

Define $U:$ LPS $\rightarrow$ Spaces to be the forgetful functor defined on objects and morphisms as follows $(M, U) \mapsto M$ and $\varphi \mapsto \varphi$.

Define $F$ : Spaces $\rightarrow$ LPS as follows. If $M$ is an object in Spaces, then let $F(M)=\left(M, \bar{M}_{\phi}\right)$, where $\bar{M}_{\phi}$ is the equivalence class of $M_{\phi}=\{M\}$ with $x \leqslant M$ $y \Longleftrightarrow x=y$. If $f: M \rightarrow N \in$ Spaces, then $F(f)=f:\left(M, \bar{M}_{\phi}\right) \rightarrow\left(N, \bar{N}_{\phi}\right)$. This is a dimap since for any $V=\left\{V_{j}\right\} \in \bar{N}_{\phi}$ with $x, y \in f^{-1} V_{j}, x \leqslant M y \Longrightarrow x=y \Longrightarrow$ $f(x)=f(y) \Longrightarrow f(x) \leqslant V_{j} f(y)$.

Remark 3.9. Note that $U$ is faithful and $F$ includes Spaces as a full subcategory of LPS.

Proposition 3.10. $F$ : Spaces $\rightleftarrows$ LPS: $U$ is an adjunction.

Proof. Let $M$ be an object in Spaces and $(N, \bar{V}) \in$ LPS. We claim that there is a natural bijection

$$
\mathbf{L P S}(F(M),(N, \bar{V})) \cong \operatorname{Spaces}(M, U(N, \bar{V})) .
$$

We need to show that there is a natural bijection

$$
\theta: \operatorname{Spaces}(M, N) \stackrel{\cong}{\longrightarrow} \mathbf{P S}\left(\left(M, \bar{M}_{\phi}\right),(N, \bar{V})\right) .
$$

If $f \in \mathbf{L P S}\left(\left(M, \bar{M}_{\phi}\right),(N, \bar{V})\right)$, then $f \in \operatorname{Spaces}(M, N)$ such that for any $V=$ $\left\{V_{j}\right\} \in \bar{V}$, for all $j,\left.f\right|_{f^{-1}\left(V_{j}\right)}$ satisfies $x \leqslant_{M} y \Longrightarrow f(x) \leqslant_{V_{j}} f(y)$. Since $x \leqslant_{M} y$ if and only if $x=y$, this last condition is vacuous. Thus, the bijection is simply $\theta: f \mapsto f$.

To show naturality, let $\alpha:(N, \bar{V}) \rightarrow\left(N^{\prime}, \bar{V}^{\prime}\right) \in$ LPS and $\xi: M^{\prime} \rightarrow M \in$ Spaces. Then

$$
\theta(U(\alpha) \circ f \circ \xi)=\alpha \circ f \circ \xi=\alpha \circ \theta(f) \circ \xi
$$

\section{The open-dicover topology}

We define the open cover Grothendieck topology for Spaces and the open dicover Grothendieck topology for LPS in the following lemma. The proof of the lemma follows directly from the definition of a basis for a Grothendieck topology.

Lemma 4.1. 1. Spaces has a Grothendieck topology whose basis is given by the open covers. For $M \in$ Spaces, let $K(M)=\{$ open covers of $M\}$. Let $J$ be the Grothendieck topology generated by $K$. Call $J$ the open cover topology. 
2. Analogously, LPS has a Grothendieck topology whose basis is given by the open dicovers in LPS. Let $K((M, \mathcal{U}))=\{$ open dicovers of $(M, \mathcal{U})\}$. Call the Grothendieck topology generated by $K$ the open-dicover topology.

In Section 3, we defined a Grothendieck topology to be subcanonical if every representable presheaf is a sheaf. In this section, we will prove that the open-dicover topology is subcanonical.

The following proposition shows that if a Grothendieck topology is generated by a basis $K$, then to see if a presheaf is a sheaf, it suffices to check the basis. For the definition of matching families and amalgamations, see Remark 2.5.

Proposition 4.2 ([MLM92, Proposition III.4.1]). Let $\mathbb{C}$ be a small category with a Grothendieck topology $J$ generated by a basis $K$. Then a presheaf $P \in \mathbf{S e t}^{\mathbb{C}^{\text {op }}}$ is a sheaf for $J$ if and only if for every $M \in \mathbb{C}$ and every cover $\left\{\phi_{j}: M_{j} \rightarrow M\right\} \in K(M)$, every matching family for $\left\{\phi_{j}\right\}$ of elements of $P$ has a unique amalgamation.

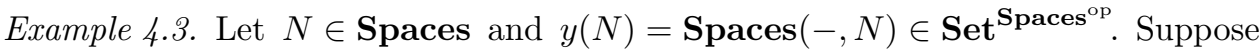
$\phi_{j}: M_{j} \rightarrow M$ is an open cover, and let $\alpha_{j}: M_{j} \rightarrow N$ be a matching family. Then $\phi_{j}$ has a unique amalgamation $\phi: M \rightarrow N$. Therefore, $y(N)$ is a sheaf for the open cover topology, and hence the open cover topology is subcanonical.

Proposition 4.4. In the open-dicover topology $J$ for local po-spaces, every representable presheaf is a sheaf. That is, $J$ is subcanonical.

Proof. Consider the representable presheaf

$$
y((N, \bar{V}))=\mathbf{L P S}(-,(N, \bar{V})) \in \mathbf{S e t}^{\mathbf{L P S}^{\text {op }}} .
$$

By Proposition 4.2, $y((N, \bar{V}))$ is a sheaf if and only if for all open dicovers $\left\{\phi_{j}\right\} \in$ $K((M, \bar{U}))$, any matching family

$$
\left\{\alpha_{j}:\left(M_{j}, \bar{U}_{j}\right) \rightarrow(N, \bar{V})\right\}
$$

has a unique amalgamation $\alpha:(M, \bar{U}) \rightarrow(N, \bar{V})$. That is, there is a map $\alpha$ such that the diagrams

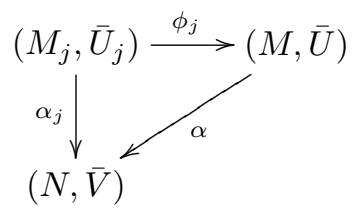

commute in LPS for all $j$.

Let $\left\{\alpha_{j}\right\}$ be such a matching family for an open dicover $\left\{\phi_{j}\right\}$. Since $\left\{\phi_{j}\right\}$ is an open dicover, then by Remark 3.7, for each $j$ there is a $U^{j}=\left\{U_{k}^{j}\right\} \in \bar{U}_{j}$ such that $U^{\prime}=\left\{U_{k}^{j}\right\}_{j, k}$ is an order atlas and $U^{\prime} \in \bar{U}$.

By definition, $\left\{\phi_{j}: M_{j} \rightarrow M\right\}$ is a cover in Spaces and $\left\{\alpha_{j}: M_{j} \rightarrow N\right\}$ is a matching family. Therefore, there is a unique amalgamation $\alpha: M \rightarrow N \in$ Spaces. That 
is, there is a map $\alpha$ such that

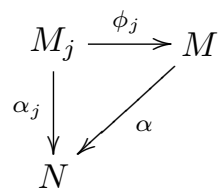

commutes in Spaces for all $j$. It remains to show that $\alpha$ is a dimap. Let $V=\left\{V_{l}\right\} \in$ $\bar{V}$. Since $\alpha_{j}:\left(M_{j}, \bar{U}_{j}\right) \rightarrow(N, \bar{V}) \in \mathbf{L P S}$, there is a $\tilde{U}^{j}=\left\{\tilde{U}_{k}^{j}\right\}_{k} \in \bar{U}^{j}$ such that for all $k, l$,

$$
\text { for all } x, y \in \tilde{U}_{k}^{j} \cap \alpha_{j}^{-1}\left(V_{l}\right), \quad x \leqslant_{\tilde{U}_{k}^{j}} y \Longrightarrow \alpha_{j}(x) \leqslant_{V_{l}} \alpha_{j}(y) .
$$

Now, for each $j$, let $\hat{U}^{j}=\left\{\hat{U}_{k}^{j}\right\}_{k} \in \bar{U}_{j}$ be a common refinement of $\tilde{U}^{j}$ and $U^{j}$. Then since $\hat{U}^{j}$ is a refinement of $\tilde{U}^{j}$,

$$
\text { for all } x, y \in \hat{U}_{k}^{j} \cap \alpha_{j}^{-1}\left(V_{l}\right), \quad x \leqslant_{\hat{U}_{k}^{j}} y \Longrightarrow \alpha_{j}(x) \leqslant_{V_{l}} \alpha_{j}(y) \text {, }
$$

and since $\hat{U}^{j}$ is a refinement of $U^{j}$, if we define $U=\left\{\hat{U}_{k}^{j}\right\}_{j, k}$, then $U \in \bar{U}$.

Since $\alpha$ is an amalgamation of $\left\{\alpha_{j}\right\}$ in Spaces if $x \in \hat{U}_{k}^{j} \subset M$, then $\alpha(x)=\alpha_{j}(x)$ and for all $l, \hat{U}_{k}^{j} \cap \alpha_{j}^{-1}\left(V_{l}\right)=\hat{U}_{k}^{j} \cap \alpha^{-1}\left(V_{l}\right)$. Therefore, using (1) for all $k, l$,

$$
\text { for all } x, y \in \hat{U}_{k}^{j} \cap \alpha^{-1}\left(V_{l}\right), \quad x \leqslant_{\hat{U}_{k}^{j}} y \Longrightarrow \alpha(x) \leqslant V_{l} \alpha(y) \text {. }
$$

That is, $\alpha$ is a dimap. Therefore, $\alpha:(M, \bar{U}) \rightarrow(N, \bar{V})$ is a unique amalgamation of $\left\{\alpha_{j}\right\}$.

\section{Equivalence of sheaves and di-étale bundles}

In this section, $\mathbb{C}$ is either Spaces or LPS with the Grothendieck topology generated by open (di)covers.

Notation 5.1. We will use $A \underset{\text { open }}{\subset} B$ to denote that $A$ is an open subset of $B$.

Notation 5.2. Let $Z \in \mathbb{C}$ and let $F \in$ Set $^{\mathbb{C}^{\text {op }}}$. Choose $x \in U \stackrel{\subset}{\stackrel{\complement}{e n}} Z$ and $s \in F(U)$.

Then for open subobjects of $U, L \stackrel{i}{\hookrightarrow} U$, we have $F(i): F(U) \rightarrow F(L)$ and we will use the notation

$$
\left.s\right|_{L}:=F(i)(s)
$$

Recall that $\operatorname{stalk}_{x}(F)=\operatorname{colim}_{x \in L \text { open } U} F(L)$ and $\operatorname{germ}_{x}(s)$ is the equivalence class represented by $s$ in $\operatorname{stalk}_{x}(F)$.

Definition 5.3. Given $Z \in \mathbb{C}$, a bundle over $Z$ is just a morphism $p: W \rightarrow Z \in \mathbb{C}$. An (di)étale bundle is a bundle which is a local (di)homeomorphism. That is, given $y \in W$, there is some open set $V \subset W$ such that $p(V)$ is open in $Z$ and $\left.p\right|_{V}$ is an isomorphism in $\mathbb{C}$. 
A morphism of (étale) bundles $p: W \rightarrow Z$ and $p: W^{\prime} \rightarrow Z$ is a morphism $\theta: W \rightarrow$ $W^{\prime} \in \mathbb{C}$ such that the following diagram commutes:

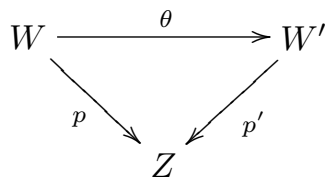

Let $\operatorname{Etale}(Z)$ denote the category of (di)étale bundles over $Z$. In addition, let $\mathcal{O}(Z)$ denote the category of open subobjects of $Z$, where the objects are open subobjects of $Z$ and the morphisms are the inclusions.

Theorem 5.4 (Theorem 1.3). Let $Z \in \mathbb{C}$. Then there is an equivalence of categories

$$
\Gamma: \operatorname{Etale}(Z) \leftrightarrows \operatorname{Shv}(\mathcal{O}(Z)): \Lambda
$$

Proof. It is well known that the statement of Theorem 1.3 is true when $\mathbb{C}=$ Spaces (see e.g. [MLM92, Corollary II.6.3]). We will show that this equivalence between étale bundles on topological spaces and sheaves on topological spaces extends to local po-spaces.

First, we describe the functors $\Gamma$ and $\Lambda$ in the case where $\mathbb{C}=$ Spaces. The functor $\Gamma$ assigns to each bundle $W \stackrel{p}{\rightarrow} Z$ the presheaf of cross-sections:

$$
\begin{aligned}
& P: \mathcal{O}(Z)^{\text {op }} \rightarrow \text { Set } \\
& U \mapsto\left\{s: U \rightarrow W \in \mathbb{C} \mid p \circ s=\operatorname{Id}_{U}\right\} \\
& U \stackrel{\theta}{\hookrightarrow} V \mapsto \theta^{*} \quad\left(\theta^{*}(t)=t \circ \theta\right) .
\end{aligned}
$$

One can check that if $p$ is étale, then $P$ is in fact a sheaf [MLM92, p. 79]. Thus, $\Gamma$ restricts to a functor $\Gamma: \operatorname{Etale}(Z) \rightarrow \operatorname{Shv}(\mathcal{O}(Z))$.

Given a presheaf $P: \mathcal{O}(Z)^{\text {op }} \rightarrow$ Set, $\Lambda(P)$ is the bundle $W \stackrel{p}{\rightarrow} Z$ where

$$
W=\left\{\operatorname{germ}_{x} s \mid x \in U \underset{\text { open }}{\subset} Z, s \in P(U)\right\} \text { and } p: \operatorname{germ}_{x} s \mapsto x .
$$

A basis for the topology on $W$ is given by the sets $\dot{s}(U)$, where $U$ is an open set in $Z, s \in P(U)$ and

$$
\begin{aligned}
\dot{s}: U & \rightarrow \Lambda(P) \\
x & \mapsto \operatorname{germ}_{x} s .
\end{aligned}
$$

Using this topology, $p: W \rightarrow Z$ is a continuous map. Again, one can check that if $P$ is a sheaf, then $W \stackrel{p}{\rightarrow} Z$ is in fact an étale bundle [MLM92, p. 85]. So, $\Lambda$ restricts to a functor $\Lambda: \operatorname{Shv}(\mathcal{O}(Z)) \rightarrow \operatorname{Etale}(Z)$.

Now, we will show that $\Gamma$ and $\Lambda$ can be similarly defined in the case where $\mathbb{C}=$ LPS. Let $p:(W, \bar{T}) \rightarrow(Z, \bar{U})$ be an étale bundle of local po-spaces. The definition of $\Gamma$ is exactly the same: $\Gamma((W, \bar{T}) \stackrel{p}{\rightarrow}(Z, \bar{U}))$ is the sheaf of cross-sections.

Given a sheaf $P$ on a local po-space $(Z, \bar{U}), \Lambda(P)=(W \stackrel{p}{\rightarrow} Z)$ is an étale bundle of topological spaces. To extend $\Lambda$ to local po-spaces, it remains to define a local order on $W$ and show that this makes $p$ a dimap.

Lemma 5.5. $W$ has a canonical local po-space structure such that $p$ is a dimap. 
Proof. Recall that the sets $\dot{s}(U)$ defined above form a basis for the topology of $W$. Choose an order atlas $\left\{\left(U_{i}, \leqslant_{i}\right)\right\} \in \bar{U}$ for $Z$. For each open sub-po-space $V \subset U_{i}$ and each $s \in P(V), \dot{s}(V) \subset W$ is a po-space under the relation

$$
\operatorname{germ}_{x} s \leqslant_{\dot{s}(V)} \operatorname{germ}_{y} s \text { if and only } x \leqslant_{i} y .
$$

This is well-defined since $\left\{U_{i}\right\}$ is an order-atlas, and it makes $\dot{s}(V)$ a po-space since $\dot{s}: U_{i} \rightarrow \dot{s}\left(U_{i}\right)$ is a homeomorphism.

We claim that

$$
T:=\left\{\dot{s}(V) \mid V \underset{\text { open }}{\subset} U_{i}, s \in P(V)\right\}
$$

is an order atlas on $W$. First, we need to show that it is an open cover. Each of the sets is open by construction. If $U \in \mathcal{O}(Z)$ and $s \in P(U)$, consider germ ${ }_{x} s$. Since $\left\{U_{i}\right\}$ is an open cover of $Z$, for some $i, x \in U_{i}$. Let $V=U \cap U_{i}$. Then $\operatorname{germ}_{x} s=$ $\left.\operatorname{germ}_{x} s\right|_{V} \in\left(\left.s\right|_{V}\right)(V)$. Therefore, $T$ is an open cover of $W$.

Finally, we need to show that the orders are compatible. For $k=1,2$, let $V_{k} \underset{\text { open }}{\subset}$ $U_{i_{k}} \underset{\text { open }}{\subset} Z$, and $s_{k} \in P\left(V_{k}\right)$. Assume $g_{1}, g_{2} \in \dot{s}_{1}\left(V_{1}\right) \cap \dot{s}\left(V_{2}\right)$. That is, $g_{1}=$ germ $_{x_{1}} s_{1}$ $=\operatorname{germ}_{x_{1}} s_{2}$ and $g_{2}=\operatorname{germ}_{x_{2}} s_{1}=\operatorname{germ}_{x_{2}} s_{2}$. For $k=1,2$,

$$
g_{1} \leqslant \dot{s}_{k}\left(V_{k}\right) g_{2} \Longleftrightarrow x_{1} \leqslant i_{k} x_{2} \text {. }
$$

Since $\left\{U_{i}\right\}$ is an order-atlas, the order $\leqslant_{i_{1}}$ and $\leqslant_{i_{2}}$ are compatible. Therefore, the orders $\leqslant_{s_{1}\left(V_{1}\right)}$ and $\leqslant_{s_{1}\left(V_{1}\right)}$ are compatible, and $T$ is an order-atlas on $W$.

Let $\bar{T}$ be the equivalence class of order atlases of $T$. We claim that $\bar{T}$ does not depend on the choice of $U \in \bar{U}$.

Let $U, U^{\prime} \in \bar{U}$, then $U$ and $U^{\prime}$ have a common refinement $U^{\prime \prime}$. Let $T, T^{\prime}, T^{\prime \prime}$ be the corresponding order-atlases for $W$ constructed as above. We will show that $T^{\prime \prime}$ is a refinement of $T$.

Let $A \underset{\text { open }}{\subset} U_{j} \in U, s \in P(A)$ and $\operatorname{germ}_{x} s \in \dot{s}(A)$. Then there is some $U_{k}^{\prime \prime} \in U^{\prime \prime}$ such that $x \in U_{k}^{\prime \prime}$ and $U_{k}^{\prime \prime}$ is a sub-po-space of $U_{j}$. Let $A^{\prime \prime}=A \cap U_{k}^{\prime \prime}$. It follows that $\left(\left.s\right|_{A^{\prime \prime}}\right)\left(A^{\prime \prime}\right) \subset \dot{s}(A)$, and $\operatorname{germ}_{x} s=\operatorname{germ}_{x}\left(\left.s\right|_{A^{\prime \prime}}\right) \in\left(\left.s\right|_{A^{\prime \prime}}\right)\left(A^{\prime \prime}\right) \in T^{\prime \prime}$. Since $U_{k}^{\prime \prime}$ is a sub-po-space of $U_{j}$, it follows that $\left(s \dot{A}_{A^{\prime \prime}}\right)\left(A^{\prime \prime}\right)$ is a sub-po-space of $\dot{s}(A)$. Thus, $T^{\prime \prime}$ is a refinement of $T$.

Similarly, $T^{\prime \prime}$ is a refinement of $T^{\prime}$ and is hence a common refinement of $T$ and $T^{\prime}$. Therefore, $\bar{T}$ does not depend on the choice of $U \in \bar{U}$.

Finally, we will show that the projection $p: W \rightarrow Z$ given by $\operatorname{germ}_{x} s \mapsto x$ is a dimap. Let $U \in \bar{U}$ be an order-atlas on $Z$. Let $T$ be the order-atlas on $W$ constructed above from $U$. Observe that $T \in \bar{T}$, since $\bar{T}$ does not depend on the choice of $U \in \bar{U}$. Let $U_{j} \in U$, let $A \underset{\text { open }}{\subset} U_{i} \in U$, and let $s \in P(A)$. Assume that

$$
\operatorname{germ}_{x_{1}} s, \operatorname{germ}_{x_{2}} s \in \dot{s}(A) \cap p^{-1}\left(U_{j}\right) .
$$

Then $x_{1}, x_{2} \in U_{i} \cap U_{j}$. By the construction of $T$ and since $U$ is an order atlas,

$$
\operatorname{germ}_{x_{1}} s \leqslant \dot{s}(A) \operatorname{germ}_{x_{2}} s \Longleftrightarrow x_{1} \leqslant U_{i} x_{2} \Longleftrightarrow x_{1} \leqslant U_{j} x_{2} .
$$

Therefore, $\Lambda$ can be extended to local po-spaces. 
Thus, we have maps

$$
\Gamma: \operatorname{Etale}(Z) \leftrightarrows \operatorname{Shv}(\mathcal{O}(Z)): \Lambda
$$

To show that they give an equivalence of categories, we will show that for a sheaf $P$ and an étale space $W \stackrel{p}{\rightarrow} Z$, there are natural isomorphisms

$$
\epsilon_{W}: \Lambda \Gamma W \rightarrow W \text { and } \eta_{P}: P \rightarrow \Gamma \Lambda P .
$$

Recall that elements of $\Lambda \Gamma W$ are of the form $\dot{s}(x)=\operatorname{germ}_{x} s$, where $s: U \rightarrow W$ satisfies $p \circ s=\operatorname{Id}_{U}$ and $x \in U$. Define $\epsilon_{W}$ to be the map $\dot{s} x \mapsto s x$. We will show that this is an isomorphism by constructing an inverse $\theta_{W}$. Let $y \in W$ and let $x=p y$. Since $W$ is étale, there exists $y \in V \underset{\text { open }}{\complement} W$ such that $\left.p\right|_{V}: V \stackrel{\cong}{\rightrightarrows} p(V)$. Let $q=\left(\left.p\right|_{V}\right)^{-1}$. Then define $\theta_{W}(y)=\operatorname{germ}_{x} q=\dot{q} x$. Then we claim $\theta_{W}$ is an inverse for $\epsilon_{W}$. Indeed

$$
\epsilon_{W} \theta_{W} y=\epsilon_{W} \dot{q} x=q x=y
$$

Also for all $\dot{s} x \in \Lambda \Gamma W, \theta_{W} \epsilon_{W} \dot{s} x=\theta_{W} s x=\operatorname{germ}_{x} t$, where $t$ is a restriction of $s$. So $\operatorname{germ}_{x} t=\operatorname{germ}_{x} s=\dot{s} x$.

Finally, we claim that $\epsilon_{W}$ and $\theta_{W}$ are dimaps. First, choose $T=\left\{T_{k}\right\} \in \bar{T}$ and $U=\left\{U_{i}\right\} \in \bar{U}$ such that $p$ satisfies the dimap condition. From $T$, construct the canonical order atlas of the form $\{\dot{s} V\}$ for $\Lambda \Gamma W$ as in the proof of Lemma 5.5. Now, let $\dot{s} x_{1}, \dot{s} x_{2} \in \dot{s} V \cap \epsilon_{W}^{-1}\left(T_{k}\right)$. Then by construction,

$$
\dot{s} x_{1} \leqslant \dot{s} V \dot{s} x_{2} \Longleftrightarrow x_{1} \leqslant_{U_{i}} x_{2} .
$$

Since $s$ satisfies the dimap condition, this implies that $s x_{1} \leqslant T_{k} s x_{2}$ which is the same as $\epsilon_{W} \dot{s} x_{1} \leqslant T_{k} \epsilon_{W} \dot{s} x_{2}$. Thus, $\epsilon_{W}$ is a dimap. Next, let $y_{1}, y_{2} \in T_{k} \cap \theta_{W}^{-1}(\dot{s} V)=$ $T_{k} \cap \epsilon_{W}(\dot{s} V)=T_{k} \cap s V$. Then there are $x_{1}, x_{2} \in V$ such that $y_{1}=s x_{1}$ and $y_{2}=s x_{2}$. Since $p$ satisfies the dimap condition

$$
y_{1} \leqslant T_{k} y_{2} \Longrightarrow p y_{1} \leqslant U_{i} p y_{2} .
$$

But this is the same as $x_{1} \leqslant_{U_{i}} x_{2}$ which implies that $\dot{s} x_{1} \leqslant \dot{s} V \dot{s} x_{2}$. Therefore, $\theta_{W}$ is a dimap.

The proof that the morphism $\eta_{P}$ is a bijection is the same as the proof in the case of topological spaces [MLM92, Theorem II.5.1].

\section{Points}

In this section, $\mathbb{C}$ is either Spaces or LPS with the Grothendieck topology generated by open (di)covers.

Let $\mathbf{S e t}^{\mathbb{C}^{\text {op }}}$ and $\operatorname{Shv}(\mathbb{C})$ be the topoi of presheaves and sheaves on $\mathbb{C}$. Recall that the inclusion functor $i: \operatorname{Shv}(\mathbb{C}) \rightarrow$ Set $^{\mathbb{C}^{\text {op }}}$ has a right adjoint $a$ called the associated sheaf functor. Recall from Definition 2.15 that if $p$ is a point in $\operatorname{Shv}(\mathbb{C})$ and $\alpha \in$ Set $^{\mathbb{C}^{\text {op }}}$, then $\operatorname{stalk}_{p}(F)=p^{*} \circ a(\alpha)$. 
Let $Z \in \mathbb{C}$. Then $Z$ is a topological space or a local po-space and we can choose any point (in the usual sense) $x \in Z$. Define

$$
\begin{aligned}
p_{x}^{*}: \text { Set }^{\mathbb{C}^{\text {op }}} & \rightarrow \text { Set } \\
F & \mapsto \underset{x \in \operatorname{Lopen}_{\subset}}{\operatorname{colim}} F(L),
\end{aligned}
$$

where the colimit is taken over all open subsets of $Z$ containing $x$. See Remark 3.5 for a discussion of subobjects in LPS.

Given a functor $p^{*}:$ Set $^{\mathbb{C}^{\text {op }}} \rightarrow$ Set, there is an induced functor

$$
A: \mathbb{C} \stackrel{y}{\rightarrow} \text { Set }^{\mathbb{C}^{\mathrm{op}}} \stackrel{p^{*}}{\longrightarrow} \text { Set, }
$$

where $y$ is the Yoneda embedding defined on objects and morphisms by $Z \mapsto \mathbb{C}(-, Z)$ and $\varphi \mapsto \mathbb{C}(-, \varphi)$.

Given a functor $A: \mathbb{C} \rightarrow$ Set, one can define induced adjoint functors $p^{*}:$ Set $^{\mathbb{C}^{\text {op }}} \rightarrow$ Set and $p_{*}:$ Set $\rightarrow$ Set $^{\mathbb{C}^{\text {op }}}\left(p^{*}=-\otimes_{\mathbb{C}} A\right.$ and $p_{*}=\mathbb{C}(A,-)$, see $[$ MLM92, Section VII.2] ).

Definition 6.1. (i) The functor $A: \mathbb{C} \rightarrow$ Set is flat if the corresponding $p^{*}$ is left exact.

(ii) $A$ is continuous if $A$ sends each covering sieve to an epimorphic family of functions. That is, if $S$ is a covering sieve, then the family of functions $\{A(\varphi) \mid \varphi \in S\}$ is jointly surjective.

Proposition 6.2 ([MLM92, Corollary VII.5.4]). Using the correspondence above, $p$ is a point in Set $^{\mathbb{C}^{\mathrm{op}}}$ if and only if $A$ is flat. Furthermore, $p$ descends to a point in $\operatorname{Shv}(\mathbb{C})$ if and only if $A$ is flat and continuous.

Proposition 6.3. $p_{x}$ defined above descends to a point in $\mathbf{S h v}(\mathbb{C})$.

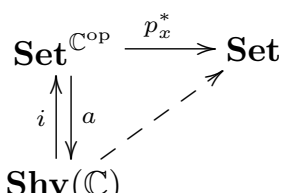

\section{$\operatorname{Shv}(\mathbb{C})$}

Proof. Let $A_{x}=p_{x}^{*} \circ y$, where $y$ is the Yoneda embedding.

First, we show that $p_{x}^{*}$ is left exact, that is, it preserves finite limits. Let $F \times_{G} H$ be a pullback in Set $^{\mathbb{C}^{\text {op }}}$.

$$
\begin{aligned}
p_{x}^{*}\left(F \times_{G} H\right) & =\operatorname{colim}_{x \in L \subseteq Z}\left(F \times_{G} H\right)(L) \\
& =\operatorname{colim}_{x \in L \subseteq Z} F(L) \times_{G(L)} H(L) \\
& =\operatorname{colim} F(L) \times_{\operatorname{colim} G(L)} \operatorname{colim} H(L) \\
& =p_{x}^{*} F \times_{p_{x}^{*} G} p_{x}^{*} H .
\end{aligned}
$$

The third equality holds because colim commutes with pullbacks in Set, and the others are by definition. Thus, $A$ is flat and $p_{x}^{*}$ is a point in Set $^{\mathbb{C}^{\text {op }}}$. 
Next, we show that $A_{x}$ is continuous. Let $\left\{Y_{i} \stackrel{\varphi_{i}}{\longrightarrow} N\right\}$ be a covering sieve for $N$ in $\mathbb{C}$. Recall that $A_{x}=p_{x}^{*} \circ y$. Let $\left(\varphi_{i}\right)_{*}$ denote composition with $\varphi_{i}$. For each arrow in the covering sieve,

$$
\begin{aligned}
p_{x}^{*} \circ y\left(Y_{i} \stackrel{\varphi_{i}}{\longrightarrow} N\right) & =p_{x}^{*}\left(\mathbb{C}\left(-, Y_{i}\right) \stackrel{\left(\varphi_{i}\right)_{*}}{\longrightarrow} \mathbb{C}(-, N)\right) \\
& =\operatorname{colim}_{x \in L \subseteq Z}\left(\mathbb{C}\left(L, Y_{i}\right) \stackrel{\left(\varphi_{i}\right)_{*}}{\longrightarrow} \mathbb{C}(L, N)\right) \\
& =y\left(Y_{i}\right)_{x} \stackrel{\left(\varphi_{i}\right)_{*}}{\longrightarrow} y(N)_{x} .
\end{aligned}
$$

We claim that this is an epimorphic family of functions in Set. Let $f \in y(N)_{x}$. Then there is an open subspace $L$ such that $x \in L \subseteq Z$ and $f$ is represented by a morphism $f^{\prime} \in \mathbb{C}(L, N)$. Since $\left\{Y_{i}\right\}$ covers $N, f^{\prime}(x) \in Y_{k}$ for some $k$. Let $K=\left(f^{\prime}\right)^{-1}\left(Y_{k}\right)$. Then $K$ is open and $x \in K \subseteq L \subseteq Z$. Furthermore, $\left.f^{\prime}\right|_{K} \in \mathbb{C}\left(K, Y_{k}\right)$ which represents an element $f^{\prime \prime} \in y\left(Y_{k}\right)_{x}$, and $\left(\varphi_{k}\right)_{*} f^{\prime \prime}=f$. Hence, we have an epimorphic family as claimed. Thus, $A$ is continuous and $p_{x}$ descends to a point in $\operatorname{Shv}(\mathbb{C})$

Abusing notation, we will also denote the induced functor in diagram (2) by $p_{x}^{*}$. With this abuse of notation, the stalk of $F \in \mathbf{S e t}^{\mathbb{C}^{\text {op }}}$ at $x$ is given by $\operatorname{stalk}_{x}(F)=$ $p_{x}^{*} a(F)=p_{x}^{*}(F)$. Note that $\operatorname{stalk}_{x}(F)=\left\{\operatorname{germ}_{x}(s) \mid x \in U \underset{\text { open }}{\subset} Z, s \in F(U)\right\}$.

Theorem 6.4. The points $p_{x}$ defined above provide enough points for $\operatorname{Shv}(\mathbb{C})$. That is, given $f \neq g: P \rightarrow Q \in \operatorname{Shv}(\mathbb{C})$, there is a $Z \in \mathbb{C}$ and a $x \in Z$ such that $p_{x}^{*} f \neq$ $p_{x}^{*} g: p_{x}^{*} P \rightarrow p_{x}^{*} Q \in$ Set.

Proof. Given $Z \in \mathbb{C}$ and either $P \in \operatorname{Shv}(\mathbb{C})$ or $f \in \operatorname{Mor} \operatorname{Shv}(\mathbb{C})$, let $P_{Z}$ or $f_{Z}$ denote the restriction to $\operatorname{Shv}(\mathcal{O}(Z))$.

Assume that $f \neq g: P \rightarrow Q \in \operatorname{Shv}(\mathbb{C})$. Thus, there is some $Z \in \mathbb{C}$ such that $f_{Z} \neq$ $g_{Z}: P_{Z} \rightarrow Q_{Z} \in \operatorname{Shv}(\mathcal{O}(Z))$.

By Theorem 1.3, this is equivalent to saying that the corresponding maps between étale spaces are not equal. That is,

$$
\Lambda f_{Z} \neq \Lambda g_{Z}: \Lambda P_{Z} \rightarrow \Lambda Q_{Z} \in \operatorname{Etale}(Z)
$$

Thus, there is some point $y \in \Lambda P_{Z}$ such that $\Lambda f_{Z}(y) \neq \Lambda g_{Z}(y)$.

By the definition of $\Lambda, y=\operatorname{germ}_{x} s$ for some $x \in U \underset{\text { open }}{\subset} Z$ and $s \in P_{Z}(U)$. That is, $y \in \operatorname{stalk}_{x}(P)=p_{x}^{*} P$. Therefore, $p_{x}^{*} f \neq g_{x}^{*} g: p_{x}^{*} P \rightarrow p_{x}^{*} Q$.

\section{Stalkwise Equivalences}

Let $(\mathbb{C}, \tau)$ be a site with a subcanonical Grothendieck topology such that $\mathbf{S h v}(\mathbb{C})$ has enough points and let $\bar{y}: \mathbb{C} \rightarrow$ sSet $^{\mathbb{C}^{\text {op }}}$ be the Yoneda embedding. Recall the definition of stalkwise equivalence in Definition 2.19 which uses the simplicial stalk functor $(\cdot)_{p}$. Also recall the Yoneda embedding $\bar{y}: \mathbb{C} \rightarrow \mathbf{s S e t}^{\mathbb{C}^{\text {op }}}$ given in Definition 2.17. Let $\varphi: X \rightarrow Y \in \mathbb{C}$.

Lemma 7.1. $\bar{y}(\varphi)$ is a stalkwise equivalence if and only if for all points $p$ in $\mathbf{S h v}(\mathbb{C})$, $p^{*} a y(\varphi) \in$ Set is an isomorphism. 
Proof. Let $p$ be a point in $\operatorname{Shv}(\mathbb{C})$. Recall that the simplicial stalk of $\bar{y}(\varphi)$ at $p$ is given by

$$
(\bar{y}(\varphi))_{p}=\left\{\operatorname{stalk}_{p}\left(\bar{y}(\varphi)_{n}\right)\right\}_{n \geqslant 0}=\left\{p^{*} a y(\varphi)\right\}_{n \geqslant 0},
$$

which is simplicially constant. Thus, $\bar{y}(\varphi)_{p} \in$ sSet is an isomorphism if and only if $p^{*} a y(\varphi) \in$ Set is an isomorphism.

Lemma 7.2. If the Grothendieck topology $\tau$ is subcanonical, then the composite functor $\mathbb{C} \stackrel{y}{\rightarrow} \mathbf{S e t}^{\mathbb{C}^{\mathrm{op}}} \stackrel{a}{\rightarrow} \mathbf{S h v}(\mathbb{C})$ is faithful.

Proof. By the Yoneda Lemma, $y$ is full and faithful. Since $\tau$ is subcanonical im $(y) \subset$ $\operatorname{Shv}(\mathbb{C})$. Furthermore, $a \circ i: \operatorname{Shv}(\mathbb{C}) \rightarrow \operatorname{Shv}(\mathbb{C})$ is naturally isomorphic to the identity functor [MLM92, Corollary III.5.6]. Thus, ay is naturally isomorphic to $y$ which is faithful.

Theorem 7.3. Let $\varphi: X \rightarrow Y \in \mathbb{C}$ and assume that $\bar{y}(\varphi)$ is a stalkwise equivalence. Then $\varphi$ is bijective.

The proof of this theorem is split into the following two propositions.

Proposition 7.4. Let $\varphi: X \rightarrow Y \in \mathbb{C}$ and assume that $\bar{y}(\varphi)$ is a stalkwise equivalence. Then $\varphi$ is epi.

Proof. For $i=1,2$, let $\psi_{i}: Y \rightarrow Z \in C$ be a morphism such that $\psi_{1} \circ \varphi=\psi_{2} \circ$ $\varphi: X \rightarrow Z$. Then for all points $p$ in $\operatorname{Shv}(\mathbb{C}), p^{*} a y\left(\psi_{1} \circ \varphi\right)=p^{*} a y\left(\psi_{2} \circ \varphi\right)$. From this, it follows that

$$
p^{*} a y\left(\psi_{1}\right) \circ p^{*} a y(\varphi)=p^{*} a y\left(\psi_{2}\right) \circ p^{*} a y(\varphi) .
$$

But by Lemma 7.1, $p^{*} a y(\varphi)$ is a set isomorphism, so, in particular, it is epi. Therefore, $p^{*} a y \psi_{1}=p^{*} a y \psi_{2}$ for all points $p$ in $\operatorname{Shv}(\mathbb{C})$. Since $\mathbb{C}$ has enough points, $a y \psi_{1}=a y \psi_{2}$. By Lemma 7.2, $a \circ y$ is faithful, and thus $\psi_{1}=\psi_{2}$. Therefore, $\varphi$ is epi.

Proposition 7.5. Let $\varphi: X \rightarrow Y \in \mathbb{C}$ and assume that $\bar{y}(\varphi)$ is a stalkwise equivalence. Then $\varphi$ is mono.

Proof. For $i=1,2$, let $\psi_{i}: W \rightarrow X \in C$ be a morphism such that $\varphi \circ \psi_{1}=\varphi \circ$ $\psi_{2}: W \rightarrow Y$. As in the proof of the previous proposition, for all points $p$ in $\operatorname{Shv}(\mathbb{C})$,

$$
p^{*} a y(\varphi) \circ p^{*} a y\left(\psi_{1}\right)=p^{*} a y(\varphi) \circ p^{*} a y\left(\psi_{2}\right) .
$$

Again by Lemma 7.1, $p^{*} a y(\varphi)$ is mono. Therefore, $p^{*} a y \psi_{1}=p^{*} a y \psi_{2}$ for all points $p$ in $\operatorname{Shv}(\mathbb{C})$. Since $\mathbb{C}$ has enough points, $a y \psi_{1}=a y \psi_{2}$. By Lemma 7.2, $a \circ y$ is faithful, thus $\psi_{1}=\psi_{2}$. Therefore, $\varphi$ is mono.

Let $\mathbb{C}=$ Spaces or LPS with the open cover topology. By Example 4.3 and Proposition 4.4, this topology is subcanonical. 
Recall from Section 6 that if $Z \in \mathbb{C}$ and $x \in Z$, then

$$
\begin{aligned}
p_{x}^{*}: \text { Set }^{\mathbb{C}^{\mathrm{op}}} & \rightarrow \text { Set } \\
F & \mapsto \underset{x \in \operatorname{copen}_{\subset}}{\operatorname{colim}} F(L)
\end{aligned}
$$

descends to a point in $\operatorname{Shv}(\mathbb{C})$ (where the colimit is taken over open subspaces of $Z$ which contain $x$ ).

Theorem 7.6. Let $\varphi: X \rightarrow Y \in \mathbb{C}$. Then $\bar{y}(\varphi)$ is a stalkwise equivalence if and only if $\varphi$ is an isomorphism in $\mathbb{C}$.

Proof. $(\Leftarrow)$ If $\varphi$ is an isomorphism, then for all points $p$ in $\operatorname{Shv}(\mathbb{C}) p^{*} a y(\varphi)$ is an isomorphism. Hence, by Lemma $7.1, \bar{y}(\varphi)$ is a stalkwise equivalence.

$(\Rightarrow)$ Assume that $\bar{y}(\varphi)$ is a stalkwise equivalence. Then by Theorem $7.3, \varphi$ is a bijection.

Let $x \in Y$. Let $p_{x}$ be the corresponding point defined in (3). Then

$$
p_{x}^{*} a y(\varphi): \operatorname{colim}_{x \in L \subseteq Y} \mathbb{C}(L, X) \stackrel{\varphi_{*}}{\longrightarrow} \operatorname{colim}_{x \in L \subseteq Y} \mathbb{C}(L, Y) \in \text { Set }
$$

is a bijection. Let $f: Y \rightarrow Y$ be given by $f=\operatorname{Id}_{Y}$. Let $\bar{f}=[f] \in \operatorname{colim}_{x \in L \subseteq Y} \mathbb{C}(L, Y)$. Let $\bar{g}=\left(\varphi_{*}\right)^{-1}(\bar{f})$. Then there is some $x \in W \subseteq Y$ such that $\bar{g}$ has a representative $g \in \mathbb{C}(W, X)$.

Let $f^{\prime}=\varphi_{*} g=\varphi \circ g$. Then $\left[f^{\prime}\right]=\varphi_{*}[g]=[f]$. Therefore, there exists $x \in S \subseteq Y$ such that $S \subset Y \cap W$ and $\left.f^{\prime}\right|_{S}=\left.f\right|_{S}=\left.\operatorname{Id}_{Y}\right|_{S}$.

Let $\psi=\left.g\right|_{S}$. Therefore, $\varphi \psi=\operatorname{Id}_{S}$. Let $T=\operatorname{im}(\psi)$. Then $\left.\varphi\right|_{T} \circ \psi=\operatorname{Id}_{S}$ and $\left.\varphi\right|_{T}$ is a bijection. Hence, $\left.\varphi\right|_{T}: T \rightarrow S$ is an isomorphism, where $x \in S$.

Finally, this construction can be repeated for all $x \in Y$. For each $x \in Y$, there is a $x \in S_{x} \subseteq Y$ and there is a map

$$
\psi_{x}: S_{x} \rightarrow X \text { such that } \psi_{x}=\left(\left.\varphi\right|_{\operatorname{im}\left(\psi_{x}\right)}\right)^{-1} .
$$

Since $\varphi$ is a bijection, all local inverses must agree. That is, $\left\{\psi_{x}: S_{x} \rightarrow X\right\}$ is a matching family on the open cover $\left\{S_{x}\right\}$ of $Y$. Since the topology is subcanonical, there is a unique amalgamation $\psi: Y \rightarrow X$. It remains to be shown that $\psi$ is an inverse for $\varphi$.

$$
\text { For all } S_{x},\left.\quad \varphi \circ \psi\right|_{S_{x}}=\varphi \circ \psi_{x}=\operatorname{Id}_{S_{x}} .
$$

Therefore, $\varphi$ is an isomorphism in $\mathbb{C}$.

\section{Model Categories for Local Po-spaces}

\subsection{A model category for local po-spaces}

Using our results on LPS, Theorem 1.1 will now follow directly from Jardine's model structure (Theorem 2.20).

Proof of Theorem 1.1. The open dicovers induce a Grothendieck topology on the small category LPS. Applying Theorem 6.4, the Grothendieck topos Shv(LPS) has enough points. So by Jardine's Theorem (Theorem 2.20), sPre(LPS) has a proper, simplicial, cellular model structure in which 
- the cofibrations are the monomorphisms, i.e. the levelwise monomorphisms of presheaves,

- the weak equivalences are the stalkwise equivalences, and

- the fibrations are the morphisms which have the right lifting property with respect to all trivial cofibrations.

Finally, by Theorem 7.6, the weak equivalences coming from LPS (via the Yoneda embedding) are precisely the isomorphisms.

\subsection{Localization}

Our main motivation for constructing a model category for local po-spaces was to model concurrent systems. In particular, we would like to be able to define and understand equivalences of concurrent systems using such a model category. However, our model structure on sPre(LPS) does not have any non-trivial equivalences among the morphisms coming from LPS. To obtain a model category more directly useful for studying concurrency, we need to localize with respect to a set of morphisms. In particular, we want morphisms which preserve certain computer-scientific information.

How to best choose such morphisms is an important question and has been studied in [Bub04]. For the sake of simplicity, that paper studied only the category PoSpaces of po-spaces (a subcategory of LPS). There it was shown that the set of morphisms which should be equivalences depends on the context. That is, instead of choosing equivalences for PoSpaces, one should be choosing equivalences for the coslice category or undercategory $\mathbf{A} \downarrow$ PoSpaces of po-spaces under a po-space $A$, where $A$ is called the context.

This result can be easily extended to our setting. First, we remark that if we choose a local po-space $A$, then the undercategory A $\downarrow$ LPS is the category whose objects are dimaps $\iota_{M}: A \rightarrow(M, \bar{U})$ and whose morphisms are dimaps $f:(M, \bar{U}) \rightarrow$ $(N, \bar{V})$ such that the following diagram commutes:

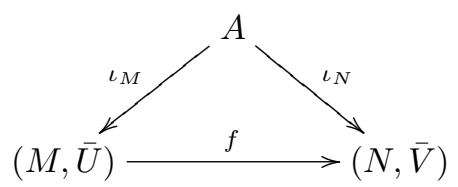

Next, $\bar{y}(A) \in \mathbf{s P r e}(\mathbf{L P S})$ and the undercategory $\overline{\mathbf{y}}(\mathbf{A}) \downarrow \mathbf{s P r e}(\mathbf{L P S})$ is the category whose objects are morphisms of simplicial presheaves $\iota_{\alpha}: \bar{y}(A) \rightarrow \alpha$ and whose morphisms are morphisms of simplicial presheaves $f: \alpha \rightarrow \beta$ such that the following diagram commutes:

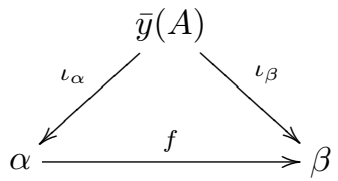

Since $\bar{y}: \mathbf{L P S} \rightarrow \mathbf{s P r e}(\mathbf{L P S})$ is a functor

$$
\bar{y}\left(\iota_{M}\right): \bar{y}(A) \rightarrow \bar{y}(M, \bar{U}) \text { and } \bar{y}\left(\iota_{N}\right)=\bar{y}\left(f \circ \iota_{M}\right)=\bar{y}(f) \circ \bar{y}\left(\iota_{M}\right) .
$$


Hence, $\mathbf{A} \downarrow$ LPS embeds as a subcategory of $\overline{\mathbf{y}}(\mathbf{A}) \downarrow \mathbf{s P r e}(\mathbf{L P S})$.

Define morphisms in $\overline{\mathbf{y}}(\mathbf{A}) \downarrow$ sPre(LPS) to be weak equivalences, cofibrations and fibrations if and only if they are weak equivalence, cofibrations and fibrations in sPre(LPS). Then this makes $\overline{\mathbf{y}}(\mathbf{A}) \downarrow \mathbf{s P r e}(\mathbf{L P S})$ into a model category (see [Hir03, Theorem 7.6.5]).

We will show that this model category is again proper and cellular. We will need the following definitions and a theorem of Kan.

Definition 8.1. (i) Let $\mathbb{C}$ be a category and $I$ be a set of maps in $\mathbb{C}$. A relative $I$-cell complex is a map that can be constructed by a transfinite composition of pushouts of elements of $I$.

(ii) An object $A \in \mathbb{C}$ is small relative to a collection of morphisms $\mathcal{D}$ in $\mathbb{C}$ if there exists a cardinal $\kappa$ such that for all regular cardinals $\lambda \geqslant \kappa$ and for all $\lambda$ sequences

$$
X_{0} \rightarrow X_{1} \rightarrow X_{2} \rightarrow \ldots \rightarrow X_{\beta} \rightarrow \ldots
$$

with $X_{\beta} \rightarrow X_{\beta+1}$ in $\mathcal{D}$ for $\beta+1<\lambda$, the set map

$$
\underset{\beta<\lambda}{\operatorname{colim}} \mathbb{C}\left(A, X_{\beta}\right) \rightarrow \mathbb{C}\left(A, \underset{\beta<\lambda}{\operatorname{colim}} X_{\beta}\right)
$$

is an isomorphism.

Definition 8.2. A model category $\mathcal{M}$ is cofibrantly generated if there are sets $I$ and $J$ such that

(i) the domains of $I$ are small relative to the relative $I$-cell complexes,

(ii) the domains of $J$ are small relative to the relative $J$-cell complexes,

(iii) the fibrations have the right lifting property with respect to $J$, and

(iv) the trivial fibrations have the right lifting property with respect to $I$.

We say that $\mathcal{M}$ is cofibrantly generated by $I$ and $J$.

Definition 8.3. (i) Let $\mathcal{M}$ be a model category cofibrantly generated by $I$ and $J$. An object $A \in \mathcal{M}$ is compact if there is a cardinal $\gamma$ such that for all relative $I$-cell complexes $f: X \rightarrow Y$ with a particular presentation, every map $A \rightarrow Y$ factors through a subcomplex of size at most $\gamma$.

(ii) $f: A \rightarrow B$ is an effective monomorphism if $f$ is the equalizer of the inclusions $B \rightrightarrows B \amalg_{A} B$.

Definition 8.4. A cellular model category is a model category cofibrantly generated by $I$ and $J$ such that

(i) the domains and codomains of elements of $I$ and $J$ are compact,

(ii) the domains of elements of $J$ are small relative to relative $I$-cell complexes, and

(iii) the cofibrations are effective monomorphisms.

Theorem 8.5 ([Hir03, Theorem 11.3.2]). Let $\mathcal{M}$ be a model category cofibrantly generated by the sets $I$ and $J$, and let $\mathcal{N}$ be a bicomplete category such that there 
exists a pair of adjoint functors $F: \mathcal{M} \leftrightarrows \mathcal{N}: U$. Define $F I=\{F u \mid u \in I\}$ and $F J=\{F v \mid v \in J\}$. If

1. the domains of FI and FJ are small relative to FI-cell and FJ-cell, respectively, and

2. U maps relative FJ-cell complexes to weak equivalences,

then $\mathcal{N}$ has a model category structure cofibrantly generated by FI and FJ such that $f$ is a weak equivalence in $\mathcal{N}$ if and only if $U f$ is a weak equivalence in $\mathcal{M}$, and $(F, U)$ is a Quillen pair.

Theorem 8.6. Let $\mathcal{M}$ be a model category and let $A \in \mathcal{M}$. Then $\mathbf{A} \downarrow \mathcal{M}$ has a model structure where a morphism

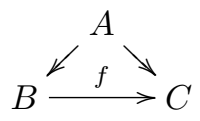

is a weak equivalence, cofibration or fibration in $\mathbf{A} \downarrow \mathcal{M}$ if and only if $f$ is a weak equivalence, cofibration or fibration, respectively, in $\mathcal{M}$. If $\mathcal{M}$ is proper, cofibrantly generated or cellular, then so is $\mathbf{A} \downarrow \mathcal{M}$.

Remark 8.7. For a more detailed proof, we invite the reader to regard Hirschhorn's note [Hir05].

Proof. That $\mathbf{A} \downarrow \mathcal{M}$ has the stated model structure follows from the definitions (see [Hir03, Theorem 7.6.5]).

Pushouts and pullbacks in $\mathbf{A} \downarrow \mathcal{M}$ can be formed by taking pushouts and pullbacks of the underlying morphisms in $\mathcal{M}$, and then taking the induced maps from $A$. It thus follows that if $\mathcal{M}$ is proper, so is $\mathbf{A} \downarrow \mathcal{M}$.

Assume $\mathcal{M}$ is cofibrantly generated by $I$ and $J$. The method for showing that A $\downarrow \mathcal{M}$ is cofibrantly generated will be to apply Theorem 8.5 to the following adjoint functors:

$$
F: \mathcal{M} \leftrightarrows(\mathbf{A} \downarrow \mathcal{M}): U
$$

where for $B \in \mathcal{M}$ and $f: B \rightarrow C \in \mathcal{M}$,

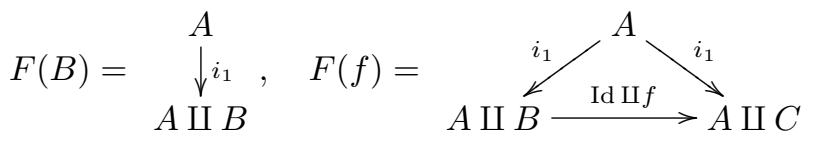

and $U$ is the forgetful functor

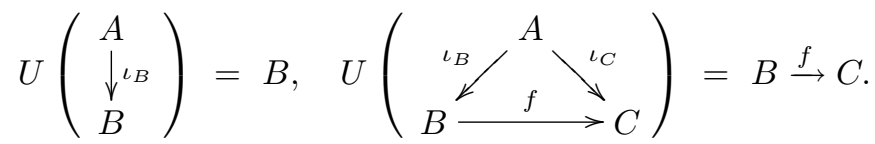

Define $F I=\{F u \mid u \in I\}$ and $F J=\{F v \mid v \in J\}$. 
The main observation for the proof is that for a morphism $u$ in $\mathcal{M}$, the pushout of $F u$ is obtained from the pushout of $u$ in $\mathcal{M}$. That is,
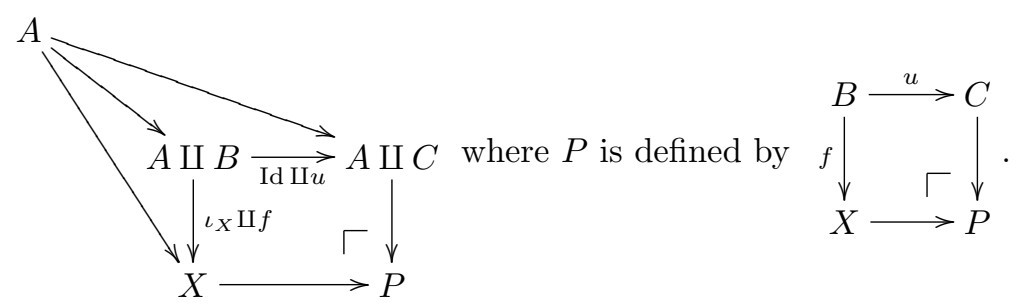

From this, it follows that for a set of morphisms $S$ in $\mathcal{M}$, the underlying morphisms of a relative $F S$-complex are a relative $S$-complex.

Hence, the conditions on $\mathbf{A} \downarrow \mathcal{M}$ in Theorem 8.5 and the definition of a cellular model category (Definition 8.4) are all inherited from the corresponding conditions in $\mathcal{M}$.

Finally, one can check that the model category structure given by Theorem 8.5 coincides with the one in the statement of the theorem.

Let $\mathcal{M}$ denote the model structure above on $\overline{\mathbf{y}}(\mathbf{A}) \downarrow \operatorname{sPre}(\mathbf{L P S})$. Since $\mathcal{M}$ is cellular, we can apply left Bousfield localization [Hir03] to this model structure $\mathcal{M}$ with respect to a set of morphisms which will preserve the computer-scientific properties we are interested in. In [Bub04], one inverted the set of dihomotopy equivalences in $\mathbf{A} \downarrow$ PoSpaces. So, in our setting, we will let $I$ be the set of dihomotopy equivalences in $\mathbf{A} \downarrow \mathbf{L P S}$ defined below. We will invert the set $\mathcal{I}=\{\bar{y}(f) \mid f \in$ $I\} \subset \overline{\mathbf{y}}(\mathbf{A}) \downarrow \mathbf{s P r e}(\mathbf{L P S})$.

Definition 8.8. (i) Let $\vec{I}$ be the po-space $([0,1], \leqslant)$ where $\leqslant$ is the usual total order on $[0,1]$. Given dimaps $f, g:(M, \bar{U}) \rightarrow(N, \bar{V}) \in \mathbf{A} \downarrow \mathbf{L P S}, \phi$ is a dihomotopy from $f$ to $g$ if $\phi:(M, \bar{U}) \times \vec{I} \rightarrow(N, \bar{V}),\left.\phi\right|_{(M, \bar{U}) \times\{0\}}=f,\left.\phi\right|_{(M, \bar{U}) \times\{1\}}=g$, and for all $a \in A, \phi\left(\iota_{M}(a), t\right)=\iota_{N}(a)$. In this case, write $\phi: f \rightarrow g$.

(ii) The symmetric, transitive closure of dihomotopy is an equivalence relation. Write $f \simeq g$ if there is a chain of dihomotopies $f \rightarrow f_{1} \leftarrow f_{2} \rightarrow \ldots \leftarrow f_{n} \rightarrow g$.

(iii) A dimap $f:(M, \bar{U}) \rightarrow(N, \bar{V})$ is a dihomotopy equivalence if there is a dimap $g:(N, \bar{V}) \rightarrow(M, \bar{U})$ such that $g \circ f \simeq \operatorname{Id}_{M}$ and $f \circ g \simeq \operatorname{Id}_{N}$.

The left Bousfield localization of $\mathcal{M}$ with respect to $\mathcal{I}$ provides a model structure on $\overline{\mathbf{y}}(\mathbf{A}) \downarrow \mathbf{s P r e}(\mathbf{L P S})$ in which the weak equivalences are the $\mathcal{I}$-local equivalences (see $[$ Hir03]), the cofibrations are the cofibrations in $\mathcal{M}$ and the fibrations are morphisms which have the right lifting property with respect to morphisms which are both cofibrations and $\mathcal{I}$-local equivalences.

Theorem 8.9 (Theorem 1.2). Let $\mathcal{I}=\{\bar{y}(f) \mid f$ is a directed homotopy equivalence rel $A\}$. The category $\overline{\mathbf{y}}(\mathbf{A}) \downarrow \mathbf{s P r e}(\mathbf{L P S})$ has a left proper, cellular model structure in which

- the cofibrations are the monomorphisms,

- the weak equivalences are the $\mathcal{I}$-local equivalences, and 
- the fibrations are those morphisms which have the right lifting property with respect to monomorphisms which are $\mathcal{I}$-local equivalences.

We claim that this model category provides a good model for studying concurrency. An analysis of this model category will be the subject of future research.

\section{Appendix A. Hypercovers}

Suppose now $\mathbb{C}$ is small and equipped with a Grothendieck topology, i.e. we have a site $(\mathbb{C}, \tau)$. The Cech structure $\mathbf{s S e t}_{\check{c}(\tau)}^{\mathbb{C}^{o p}}$ is obtained from the projective structure by homotopically localizing the comparison morphisms given by the Čech covers with respect to $\tau$ or, up-to homotopy, from the injective structure by localizing at the same set of morphisms.

Definition A.1. Let $U=\left\{U_{i} \stackrel{u_{i}}{\longrightarrow} X\right\}_{i \in I} \in J(X)$ be a cover. Let $i_{p} \in I$ for each $0 \leqslant p \leqslant n$ and $U_{i_{0} \ldots i_{n}}$ be the wide pullback of the $u_{i_{p}}$ 's, i.e. the limiting object of the diagram

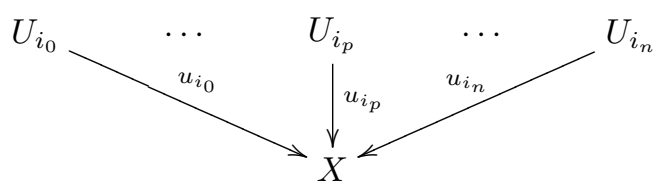

The $\check{C} e c h$ nerve $\check{U}$ of $U$ is the simplicial presheaf given by

$$
\check{U}_{n} \stackrel{\text { def }}{=} \coprod_{i_{0}, \ldots, i_{n} \in I} y\left(U_{i_{0} \ldots i_{n}}\right)
$$

Remark A.2. For any $n \in \mathbb{N}, X \in \mathbb{C}$ and $U \in J(X)$, there is a morphism

$$
u_{i_{0} \cdots i_{n}}: U_{i_{0} \ldots i_{n}} \rightarrow X
$$

and a diagram of presheaves

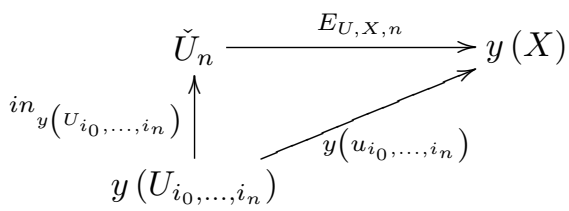

where $E_{U, X, n}$ is given by universal property. The $E_{U, X, n}$ assemble to a morphism of simplicial presheaves

$$
E_{U, X}: \check{U} \rightarrow \kappa_{y(X)}
$$

Remark A.3. Given $U \in J(X)$ seen as a subcategory of the slice $\mathbb{C} / X$, there is the evident functor

$$
\begin{aligned}
& \delta_{U}: \quad U \rightarrow \text { sSet }^{\mathbb{C}^{o p}} \\
& u_{i} \mapsto \kappa_{y\left(U_{i}\right)}
\end{aligned}
$$


Proposition A.4. Localizing $\mathbf{s S e t}_{i n j}^{\mathbb{C}^{o p}}$ at the sets

(i) $\left\{E_{U, X} \mid X \in \mathbb{C}, U \in J(X)\right\}$;

(ii) $\left\{\operatorname{hocolim}\left(\delta_{U}\right) \rightarrow \kappa_{y(X)} \mid X \in \mathbb{C}, U \in J(X)\right\}$;

(iii) $\left\{\kappa\left(\iota_{U}\right) \mid X \in \mathbb{C}, U \in J(X)\right\}$, where, given $X \in \mathbb{C}$ and $R$ a sieve on $X$, $\iota_{R}: R \hookrightarrow y(X)$ is the corresponding inclusion of presheaves;

(iv) $\left\{\eta_{F}: F \rightarrow j(F) \mid F \in \mathbf{s S e t}^{\mathbb{C}^{o p}}\right\}$, where $j: \mathbf{s S e t}^{\mathbb{C}^{o p}} \rightarrow \mathbf{s S e t}^{\mathbb{C}^{o p}}$ is the objectwise sheafification functor;

yields the same model structure $\mathbf{s S e t}_{\breve{c}(\tau)}^{\mathbb{C}^{o p}}$. The same holds for the projective version.

Finally, there is a model structure $\mathbf{s S e t}_{h y p(\tau)}^{\mathbb{C}^{o p}}$ obtained from the projective structure by homotopically localizing at the set of the comparison morphisms given by hypercovers with respect to $\tau$. This model structure is Quillen equivalent to Jardine's model structure (Theorem 2.20) on $\mathbf{s S e t}^{\mathbb{C}^{\mathrm{op}}}$ [DHIO4, Theorem 1.2]. As with the Čech structure, there is also an injective version. Since Cech covers are particular hypercovers, there is the series of inclusions

$$
\mathcal{W}_{\text {prj }} \subseteq \mathcal{W}_{\check{c}(\tau)} \subseteq \mathcal{W}_{\text {hyp }(\tau)}
$$

and a similar series for the injective version. It is in general the case that $\mathcal{W}_{\check{c}(\tau)} \varsubsetneqq$ $\mathcal{W}_{\text {hyp }(\tau)}$, yet equality holds in some important particular cases like the smooth Nisnevitch site (c.f. [DHI04, Example A10]). It is an interesting question whether or not $\mathcal{W}_{\check{c}(\tau)}=\mathcal{W}_{h y p(\tau)}$ for local po-spaces.

\section{References}

[AGV72] M. Artin, A. Grothendieck, and J. L. Verdier. Théorie des Topos et Cohomologie Etale des Schémas, Lecture Notes in Math., Vols. 269 and 270, Springer-Verlag, Berlin, 1972.

[BK72] A. K. Bousfield and D. M. Kan. Homotopy limits, completions and localizations. Lecture Notes in Math., Vol. 304, Springer-Verlag, Berlin, 1972.

[Bub04] P. Bubenik. Context for models of concurrency. In: Prelimary Proceedings of the Workshop on Geometry and Topology in Concurrency and Distributed Computing GETCO 2004, Vol. NS-04-2 of BRICS Notes, BRICS, Amsterdam, The Netherlands, (2004) pp. 33-49. Also to appear in Electron. Notes Theor. Comput. Sci.).

[DHI04] D. Dugger, S. Hollander, and D. C. Isaksen. Hypercovers and simplicial presheaves. Math. Proc. Cambridge Philos. Soc., 136(1) (2004), pp. 9 51.

[Dij68] E. W. Dijkstra. Cooperating sequential processes. Academic press, 1968.

[Dug01] D. Dugger. Universal homotopy theories. Adv. Math., 164(1) (2001), pp. 144-176.

[FGR99] L. Fajstrup, E. Goubault, and M. Raussen. Algebraic topology and concurrency. Theoret. Comput. Sci., 1999 to appear. Also preprint 
R-99-2008, Department of Mathematical Sciences, Aalborg University, Aalborg, Denmark.

[Gau03] P. Gaucher. A model category for the homotopy theory of concurrency. Homology Homotopy Appl., 5(1) (2003), pp. 549-599.

[Gou96] E. Goubault. Durations for truly-concurrent transitions. In: ESOP '96: Proceedings of the 6th European Symposium on Programming Language and Systems, Vol. 1058, Lecture Notes Comput. Sci., pp. 173-187. Springer-Verlag, London, UK, 1996.

[Gou02] E. Goubault. Labelled cubical sets and asynchronous transitions systems: an adjunction. In: Preliminary Proceedings CMCIM'02, 2002. http://www.di.ens.fr/ goubault/papers/cmcim02.ps.gz

[Gou03] E. Goubault. Some geometric perspectives in concurrency theory. Homology Homotopy Appl., 5(2) (2003), pp. 95-136 (electronic). Algebraic topological methods in computer science, Stanford, CA, 2001.

[Gra03] M. Grandis. Directed homotopy theory. I. Cah. Topol. Géom. Différ. Catég., 44(4) (2003), pp. 281-316.

[Hir03] P. S. Hirschhorn. Model categories and their localizations, Math. Surveys Monogr., 99, (2003).

[Hir05] P. S. Hirschhorn. Overcategories and undercategories of model categories. http://www-math. mit.edu/ psh/, 2005.

[Hov99] M. Hovey. Model categories, Math. Surveys Monogr., 63, (1999).

[Jar87] J. F. Jardine. Simplicial presheaves. J. Pure Appl. Algebra, 47(1) (1987), pp. 35-87.

[Jar96] J. F. Jardine. Boolean localization, in practice. Doc. Math., 1(13) (1996), pp. 245-275 (electronic).

[Joh77] P. T. Johnstone. Topos Theory. London Mathemathical Society Monographs, Academic Press, 1977.

[Joy84] A. Joyal. Homotopy theory of simplicial sheaves. unpublished (circulated as a letter to Grothendieck), 1984.

[Law63] F. W. Lawvere. Functorial Semantics of Algebraic Theories. PhD thesis, Columbia University, 1963.

[Law64] F. W. Lawvere. An elementary theory of the category of sets. Proc. Natl. Acad. Sci. USA, 52 (1964), pp. 1506-1511.

[Law73] F. W. Lawvere. Metric spaces, generalized logic, and closed categories. Rend. Sem. Mat. Fis. di Milano, 43 (1973), pp. 135-166.

[Mil80] R. Milner. A calculus of communicating systems, 92, Lect. Notes Comput. Sci.. (1980).

[MLM92] S. Mac Lane and I. Moerdijk. Sheaves in geometry and logic. In: A first introduction to topos theory. Universitext. Springer-Verlag, New York, 1992.

[Qui67] D. G. Quillen. Homotopical algebra. Lecture Notes in Math., Vol. 43, Springer-Verlag, Berlin, 1967. 
Peter Bubenik p.bubenik@csuohio.edu

Department of Mathematics

Cleveland State University

2121 Euclid Ave. RT 1515

Cleveland OH, 44115-221

USA

\author{
Krzysztof Worytkiewicz kworytki@uwo.ca \\ Department of Mathematics \\ University of Western Ontario \\ Middlesex College \\ London, Ontario N6A 5B7 \\ Canada
}

This article is available at http://intlpress.com/HHA/v8/n1/a10 